\title{
Investigating the evolution of PKS B1144-379: comparison of VLBI and scintillation techniques
}

\author{
N. M. M. Said, ${ }^{1,2 \star ~ S . ~ P . ~ E l l i n g s e n ~}{ }^{\circledR},{ }^{1 \star}$ S. Shabala ${ }^{\circledR},{ }^{1 \star}$ G. Orosz,${ }^{1}$ J. Liu ${ }^{\circledR},{ }^{3}$ H. E. Bignall, ${ }^{2}$ J. \\ N. McCallum ${ }^{1}$ and C. Reynolds ${ }^{2}$ \\ ${ }^{1}$ School of Natural Sciences, University of Tasmania, Private Bag 37, Hobart, TAS 7001, Australia \\ ${ }^{2}$ CSIRO Astronomy and Space Science, P.O. Box 1130, Bentley, WA 6102, Australia \\ ${ }^{3}$ Max-Planck-Institut für Radioastronomie, Auf dem Hügel 69, D-53121 Bonn, Germany
}

Accepted 2021 September 13. Received 2021 September 7; in original form 2020 September 27

\begin{abstract}
We have investigated the evolution of the BL Lac object PKS B1144-379 using the University of Tasmania Ceduna 30-m radio telescope at a frequency of $6.7 \mathrm{GHz}$ and very long baseline interferometry (VLBI) data at 8.6 GHz. Variability time-scales associated with two flares detected in 2005 November and 2008 August were derived from long-term variations in total flux density monitored by Ceduna between 2003 and 2011. A kinematic study of the parsec-scale jet of PKS B1144-379 was performed using VLBI data obtained between 1997 and 2018. Quasi-periodic flarings with a period of 3-4 yr were observed. Over the 20-yr interval, the average jet position angle was found to be $\sim 150^{\circ}$.
\end{abstract}

Key words: galaxies: jets - quasars: individual: PKS B1144-379.

\section{INTRODUCTION}

BL Lacertae is the prototype of the BL Lac class of active galactic nuclei (AGNs). Its nuclear region exhibits flux variability on time-scales ranging from several hours to years over the whole electromagnetic spectrum (Padovani \& Giommi 1995). This variability appears to be related to the observed morphology and kinematics of the jet plasma on parsec scales. The jet originates close to the central supermassive black hole and is directed near to the line of sight (e.g. Blandford \& Königl 1979; Urry \& Padovani 1995; Jorstad et al. 2001; Zensus et al. 2002). The jet's small inclination with respect to the line of sight also allows the observation of the Broad Line Region (BLR), which is present perpendicular to the jet (Cao 2004). Very long baseline interferometry (VLBI) monitoring observations are a useful tool to study the kinematic properties of AGN jets. Interferometric images of BL Lac objects at radio wavelengths show the presence of a compact core and a diffuse halo-like source on arcsec scales (Antonucci 1986), whereas at higher resolutions ( $\sim 10$ mas), they show a core-jet structure with components following different trajectories (Polatidis et al. 1995). Previous multi-epoch surveys of large radio-loud AGN samples (e.g. Kellermann et al. 2004; Piner et al. 2007; Britzen et al. 2008; Lister et al. 2009a; Marscher et al. 2011; Piner et al. 2012; Lister et al. 2013) have revealed complex kinematics in individual sources, including jet curvature, acceleration and deceleration, nonradial motion, stationary, or inward-moving features.

To date, it is still not clear whether AGN jets consist of a pair of plasma or a proton-electron plasma (i.e. leptonic or hadronic jets) (Böttcher et al.2015), nor do we have a comprehensive understanding

^E-mail: noor.mdsaid@utas.edu.au (NMMS); simon.ellingsen@utas.edu.au (SPE); stanislav.shabala@utas.edu.au (SS) of how they are launched and collimated. The two best-known jet launching mechanisms are the Blanford-Payne process (Blandford \& Payne 1982) and the Blandford-Znajek mechanism (Blandford \& Znajek 1977). In the Blandford-Payne process (Blandford \& Payne 1982), a magnetic field threading the accretion disc and frozen into the disc plasma is wound up by the rotation of the disc. This gives rise to a magnetic pressure gradient that accelerates material from the disc outward along the field lines. The Blandford-Znajek mechanism (Blandford \& Znajek 1977) proposes that the jets are launched from the ergosphere of the black hole by extracting the rotational energy of the black hole. The rotation of the black hole in the ambient magnetic field of the accretion disc gives rise to induced electric fields that accelerate local charged particles. As a consequence, a series of processes that give rise to jets comprised of electrons and positrons carried outward by an outgoing Poynting flux is triggered. Common factors in both mechanisms are the rotation of the central black hole and its accretion disc in the presence of a magnetic field, giving rise to a helical magnetic field carried outward along with the jets (Tateyama et al. 1998; Lovelace et al. 2002; Nakamura \& Meier 2004, 2014; Nakamura, Garofalo \& Meier 2010). Various theoretical works (e.g. Tchekhovskoy, Narayan \& McKinney 2011; Tchekhovskoy \& Bromberg 2016) have confirmed this basic picture of jet launching and propagation. However, in addition to the helical magnetic field being transported outward in the jet, several other mechanisms can affect the observed jet properties. These include shocks (Marscher \& Gear 1985), instabilities (Hardee 2011), sites of enhanced Doppler boosting due to geometrical effects (Camenzind \& Krockenberger 1992), bending of the jets (Lister 2001; Britzen et al. 2010; Gabuzda 2018), turbulence, and magnetic reconnection (Asada \& Nakamura 2012; Nakamura \& Asada 2013; Cohen et al. 2014, 2015).

In this paper, we used 6.7- GHz COSMIC [Continuous Single Dish Monitoring of Intraday Variability (IDV) at Ceduna] data taken 
between 2003 and 2011 (McCulloch et al. 2005; Blanchard 2013), and 8.6-GHz VLBI images from Radio Reference Frame Image Database (RRFID) ${ }^{1}$ and Astrogeo ${ }^{2}$ data base observed between 1997 and 2018. In a previous publication related to PKS B1144-379 (Said et al. 2020, hereafter Paper I), we give background on the published literature for this object. We also found that the $\chi^{2}$ value for the annual cycle fittings for the years 2005 and 2008 was higher than for the other observation years. This has shown that the fitted parameters have not been well constrained and are likely due to the intrinsic source evolution entangled with the interstellar scintillation (ISS) (Paper I). Our findings are consistent with the previous study by Shabala et al. (2012a), which reported that the increase in total flux density of $S(2.3 \mathrm{GHz})$ and $X(8.4 \mathrm{GHz})$ bands taken from the International VLBI Service for Geodesy and Astrometry (IVS) ${ }^{3}$ data at MJD 53200 (2004 July 14) and 54000 (2006 September 22 ) is indicative of a flare. They also found that after MJD of 53500 (2005 May 10), both $S$ - and $X$-band flux density increased, corresponding to the emergence of a new jet component (with a delay of $\sim 150 \mathrm{~d}$ ). These results lead us to divide the long-term variations in the mean flux density of PKS B1144-379 into several phases of source evolution.

This paper aims to study the source evolution and the morphology of the parsec-scale jet in the BL Lac object PKS B1144-379 and its relation to the evolution of the centimetre wavelength flux density and ISS properties. Section 2 describes the observations and data analysis procedures. In Sections 3 and 4, we present our results from the single-dish total flux density and VLBI monitoring, respectively. Section 5 discusses our findings and we conclude our discussions in Section 6. Throughout this paper, we adopt the following cosmological parameters: $H_{\mathrm{o}}=69.6 \mathrm{~km} \mathrm{~s}^{-1} \mathrm{M} \mathrm{pc}^{-1}, \Omega_{\mathrm{m}}$ $=0.286, \Omega_{\Lambda}=0.714$, and $z=1.048$.

\section{OBSERVATIONS AND DATA ANALYSIS}

\subsection{Single-dish, total flux density monitoring}

In Paper I, we focused on the single-dish measurements of PKS B1144-379 made as part of the COSMIC project (McCulloch et al. 2005; Blanchard 2013). We have utilized the same data in the current investigation and we refer the reader to Paper I for further details on the single-dish data and its analysis.

Measurements of the variability time-scales associated with two flares detected in 2005 November and 2008 August (Shabala et al. 2012a; Paper I) were determined using the Burbidge, Jones \& Odell (1974) method. The logarithmic variability time-scale $\left(\tau_{\mathrm{obs}}\right)$ is defined to be

$\tau_{\mathrm{obs}}=\mathrm{d} t / \mathrm{d}[\ln (S)]$,

where $\mathrm{d} t$ and $\mathrm{d}[\ln (S)]$ are the observation time and the natural logarithm of the flux density, respectively. We fitted a straight line to the rising and/or decreasing parts of each of the identified flares. We used the logarithmic variability time-scale to determine the brightness temperature corrected for the cosmological redshift using the following equation:

$T_{\mathrm{b}, \mathrm{obs}}=5.87 \times 10^{21} \frac{\lambda^{2} \Delta S}{\tau_{\mathrm{obs}}^{2}}\left[1-\frac{1}{\sqrt{1+z}}\right]^{2}$,

\footnotetext{
${ }^{1}$ https://www.usno.navy.mil/USNO/astrometry/vlbi-products/rrfid

${ }^{2} \mathrm{http}: / /$ astrogeo.org/vlbi-images/

${ }^{3}$ https://ivscc.gsfc.nasa.gov/products-data/data.html
}

where $z$ is the redshift, $\lambda$ is the observing wavelength in metres, $\Delta S$ is the amplitude of the flare in Jy, and the logarithmic variability time-scale $\tau_{\mathrm{obs}}$ is in days. The amplitude $\Delta S$ was calculated from the linear fit as the difference between the fitted flux density at the first and last observing epochs (Terasranta \& Valtaoja 1994). We then estimated an upper limit for the linear size of the emitting region as $R=c \tau_{\text {obs }}$, where $c$ is the speed of light and $\tau_{\text {obs }}$ is the logarithmic variability time-scale (Gorshkov et al. 2018).

\subsection{VLBI monitoring}

We have analysed 52 radio images of PKS B1144-379 taken from the RRFID and Astrogeo VLBI FITS image data base observed at $8.6 \mathrm{GHz}$ between the years 1997 and 2018 . However, it should be noted that these are snapshot data sets with limited $(u, v)$ coverage and dynamic range. We have used DIFMAP (Shepherd 1997) and its $\chi^{2}$ minimization algorithm MODELFIT to fit the complex visibility data with Gaussian components and to quantify important parameters such as the flux density, radial separation from the phase centre, and position angle (PA defined from north to east, in the counterclockwise direction). We applied simple models consisting of two elliptical Gaussian components, one representing the core and the other the inner part of a one-sided jet. In this case, two additional model parameters were fitted, the axial ratio and the position angle of the major axis. The core is the brightest part of the self-absorbed jet (e.g. Shabala, Santoso \& Godfrey 2012b), and its location changes with frequency, i.e. core shift effect (Lobanov 1998; Plavin et al. 2019). At each epoch, we determined the innermost jet position angle by taking a flux-density-weighted position angle average of all clean components above three times the image noise level from the core.

The image for each epoch was analysed independently by building the model iteratively, i.e. adding new components as long as the residual image showed significant flux density and the new component improved the overall fit in terms of the $\chi^{2}$ value. The addition of a component was followed by iterations of MODELFIT (until the fit converged) and self-calibration of the visibility phases. In addition, a cross-check of our model was performed by comparing the position of the components with the brightness distribution in the CLEAN image of DIFMAP. We used the following model fitting uncertainties. An uncertainty of 10 per cent for the component flux density is a reasonable and widely used estimate (Lister et al. 2009b, 2013). If the component is unresolved, the uncertainty in the position is set to onefifth of the beam size; otherwise, the uncertainty is estimated using one-fifth of the component full width at half-maximum (FWHM). The positional uncertainty $(\Delta X)$ in mas is converted into degrees using the simple trigonometric formula $\delta \mathrm{PA}=\arctan (\Delta X / r)$, where $r$ is the radial separation in mas (Karamanavis et al. 2016).

\section{RESULTS FROM TOTAL FLUX DENSITY MONITORING}

Fig. 1 shows the long-term variations in the mean flux density of PKS B1144-379 taken as part of the COSMIC observing project between 2003 and 2011. The mean flux density of PKS B1144-379 at $6.7 \mathrm{GHz}$ over this 9-yr observation period was found to vary from 0.8 to $3.4 \mathrm{Jy}$, with two flares in 2005 November and 2008 August (Shabala et al. 2012a; Paper I). The time interval between point A and $\mathrm{B}$ is likely to be the jet dispersion phase due to the flare that occurred in 2002 September, which was detected as the peak flux density in the VLBI 8.6-GHz data base. At this phase, the mean flux density decreased from $2.2 \mathrm{Jy}$ at point A to $0.8 \mathrm{Jy}$ at point B. Measurement of the logarithmic variability time-scale $\left(\tau_{\text {obs }}\right)$, brightness temperature 


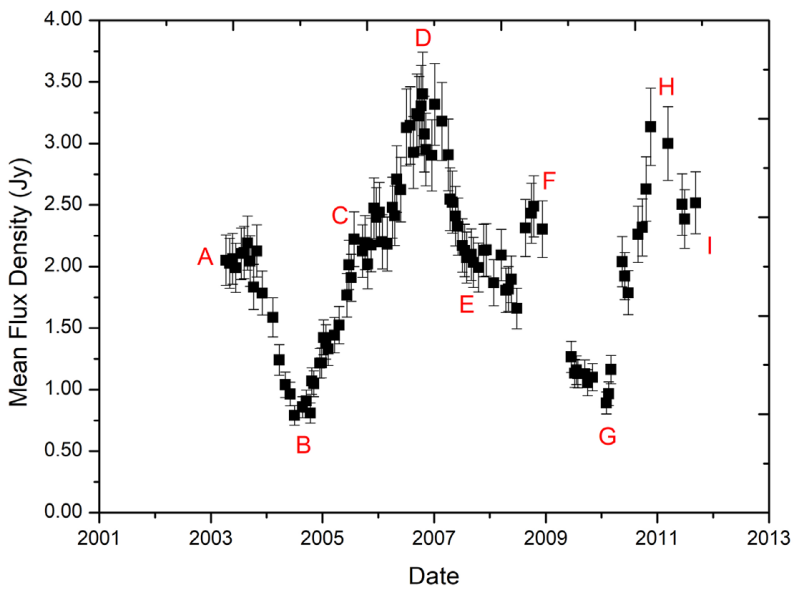

Figure 1. Long-term monitoring of variations of flux density for PKS B1144-379 between 2003 and 2011. Variations in the total flux density of the source can be divided into several phases; core brightening (points $\mathrm{B}-\mathrm{C}, \mathrm{E}-\mathrm{F}$, and $\mathrm{G}-\mathrm{H}$ ), jet expansion (points $\mathrm{C}-\mathrm{D}$ ), and jet dispersion (points A-B, D-E, F-G, and H-I). Two flares in the total flux density were detected in 2005 November (point C) and 2008 August (point F). Data were taken from the COSMIC observing project from 2003 through 2011 at $6.7 \mathrm{GHz}$.

( $\left.T_{\mathrm{b}, \mathrm{obs}}\right)$, and linear size of the emitting region $(R)$, at this phase could not be undertaken as no data were observed during the flare. Point $\mathrm{B}$ to $\mathrm{C}$ is assumed to be the core brightening phase with point $\mathrm{B}$ as the starting point for a quiescent state. The mean flux density of the core component increased from 0.8 to $2 \mathrm{Jy}$ at point $\mathrm{C}$, where the first flare was detected in 2005 November. The linear size of the emitting region was estimated to be $0.38 \mathrm{pc}$ (below the resolution available even from VLBI imaging). The observation period between point $\mathrm{C}$ and $\mathrm{D}$ is considered to be the jet expansion phase. Throughout this phase, the mean flux density increased from 2.0 to $3.4 \mathrm{Jy}$ and the linear size of the emitting region increased to $0.67 \mathrm{pc}$.

As the mean flux density decreased from its peak at D to $1.8 \mathrm{Jy}$ at $\mathrm{E}$, this implies that the jet dispersion phase began at point $\mathrm{D}$ and continued at point E. During this phase, we found that the linear size of the emitting region was reduced to $0.56 \mathrm{pc}$. Between point $\mathrm{E}$ and $\mathrm{F}$, the mean flux density increased slightly to $2.5 \mathrm{Jy}$, marking the start of a new core brightening phase (the second flare occurred in 2008 August at point F). We calculate that the linear size of the emitting region increased to $0.74 \mathrm{pc}$ during this phase. The source experienced a second jet dispersion phase (points $F-G$ ), where we observed the mean flux density and the linear size of the emitting region decreased to $0.8 \mathrm{Jy}$ and $0.37 \mathrm{pc}$ at point $\mathrm{G}$, respectively. During 2010 (points $\mathrm{G}-\mathrm{H}$ ), the mean flux density increased again to $3.0 \mathrm{Jy}$, while the linear size of the emitting region was estimated to be $0.22 \mathrm{pc}$. The increasing mean flux density suggests that there was probably another flare that occurred in 2010 November. The mean flux density then continued to decrease to point I indicating the beginning of the jet dispersion phase. Fig. 2 plots the natural logarithm of the flux density of PKS B1144-379 as a function of time, with the different colours showing different periods during the source evolution. We summarize results of the natural logarithm of the flux density of PKS B1144-379 in Table 1.

\section{RESULTS FROM VLBI MONITORING}

Fig. 3 displays the long-term variations in the core and jet flux density of PKS B1144-379 at 8.6 GHz between 1997 and 2018 . Data were taken from the VLBI data sets of RRFID and Astrogeo.

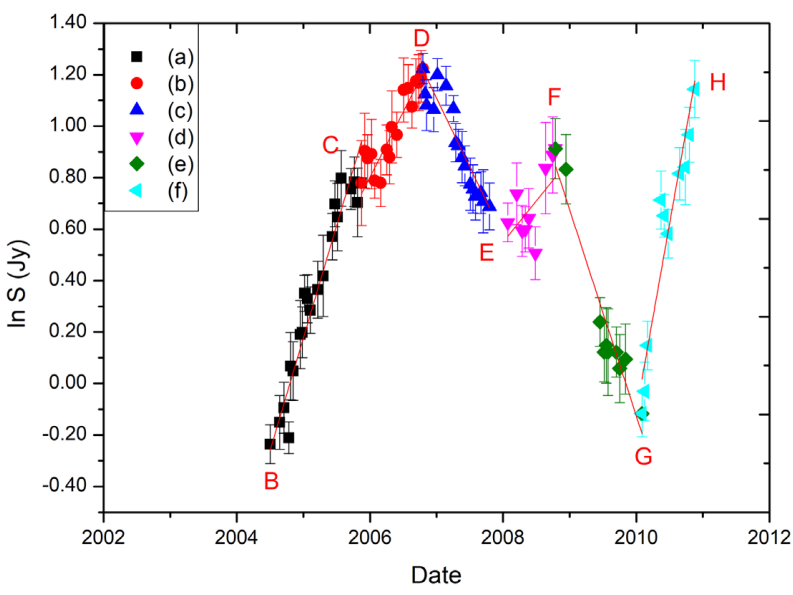

Figure 2. The total flux density of PKS B1144-379 is plotted on a logarithmic scale. The straight line in each phase is a least-squares fit to the data, where its slope determines the logarithmic variability time-scale $\tau_{\text {obs. }}$. The long-term variations in the mean flux density of this source are divided into several phases of source evolution: core brightening phase (points B-C, E-F, and $\mathrm{G}-\mathrm{H}$ ), jet expansion phase (points $\mathrm{C}-\mathrm{D}$ ), and jet dispersion phase (points $\mathrm{D}-\mathrm{E}$ and $\mathrm{F}-\mathrm{G}$ ). Data were taken from the COSMIC observing project from 2003 through 2011 at $6.7 \mathrm{GHz}$.

The core and jet flux density were found to vary from 0.84 to 2.65 and 0.02 to $0.26 \mathrm{Jy}$, respectively. Four peaks of flux density observed in 1998 April, 2002 September, 2008 December, and 2012 December were most likely due to flares. It should be noted, however, that a limited number of observing epochs may have an impact on our prediction of flares by simply inspecting the peak of the flux density, particularly for the years 2008 December and 2012 December. The jet orientation of the source was found to be mostly in the south-east direction measured using the TANAMI data (Ojha et al. 2010, fig. 3). However, over the 20-yr interval, we found that the jet measured from the brightest (core) to the second brightest (jet) component shows changes in the position angle, ranging from $125^{\circ}$ to $176^{\circ}$ with separation of the jet component varied between 0.57 and 4.96 mas, as shown by Fig. 4. MODELFIT results and the CLEAN images of PKS B1144-379 used in our analysis can be found in Table A1 and Fig. B1.

\section{DISCUSSION}

In Paper I, we used the 'Earth Orbital Synthesis' technique (Macquart \& Jauncey 2002), which utilised the extrinsic effect known as interstellar scintillation (ISS) to investigate the evolution of PKS B1144-379. We found that the entanglement between the intrinsic source evolution with the ISS is likely to result in a higher value of $\chi^{2}$ for the annual cycle fittings for the years 2005 and 2008 and an indication of flares. These results are supported by the VLBI observations reported by Shabala et al. (2012a). Shabala et al. (2012a) also detected the emergence of a new jet component after MJD of 53500 (2005 May 10) in both $S$ - and $X$-band flux density, and this result is in agreement with an increase in the linear size of the emitting region [jet expansion and jet dispersion phase; points $\mathrm{C}-\mathrm{D}(0.67 \mathrm{pc})$ and points D-E $(0.56 \mathrm{pc})$, respectively] compared to the brightening phase at points $\mathrm{B}-\mathrm{C}(0.38 \mathrm{pc})$. In Paper I, we also found that the size of the scintillating component for the years 2006 and 2007 increased from $9 \mu$ as (during the year 2004) to 13 and $15 \mu$ as, respectively. The decrease in the linear size of the emitting region during the jet dispersion ( $0.37 \mathrm{pc}$ at points $\mathrm{F}-\mathrm{G})$ and the core brightening phase 
Table 1. Natural logarithm of the flux density of PKS B1144-379 at $6.7 \mathrm{GHz}$. Columns from left- to right-hand side: (1) legend labels of Fig. 2, (2) phase, (3) and (4) logarithmic variability time-scale ( $\left.\tau_{\mathrm{obs}}\right)$ and its uncertainty, (5) and (6) brightness temperature ( $T_{\mathrm{b}, \mathrm{obs}}$ ) and its uncertainty, and (7) and (8) linear size of the emitting region $(R)$ and its uncertainty.

\begin{tabular}{lrcccccc}
\hline & Phase & $\tau_{\mathrm{obs}}(\mathrm{d})$ & $\sigma(\mathrm{d})$ & $T_{\mathrm{b}, \mathrm{obs}}\left(10^{12} \mathrm{~K}\right)$ & $\sigma\left(10^{12} \mathrm{~K}\right)$ & $R(\mathrm{pc})$ & $\sigma(\mathrm{pc})$ \\
\hline (a) & Core brightening (points B-C) & 448 & 29 & 7.40 & 1.98 & 0.38 & 0.02 \\
(b) & Jet expansion (points C-D) & 794 & 100 & 1.60 & 0.57 & 0.67 & 0.08 \\
(c) & Jet dispersion (points D-E) & 671 & 64 & 3.40 & 0.73 & 0.56 & 0.05 \\
(d) & Core brightening (points E-F) & 885 & 348 & 0.85 & 0.67 & 0.74 & 0.29 \\
(e) & Jet dispersion (points F-G) & 439 & 42 & 9.00 & 2.22 & 0.37 & 0.04 \\
(f) & Core brightening (points G-H) & 258 & 29 & 35.30 & 6.90 & 0.22 & 0.02 \\
\hline
\end{tabular}

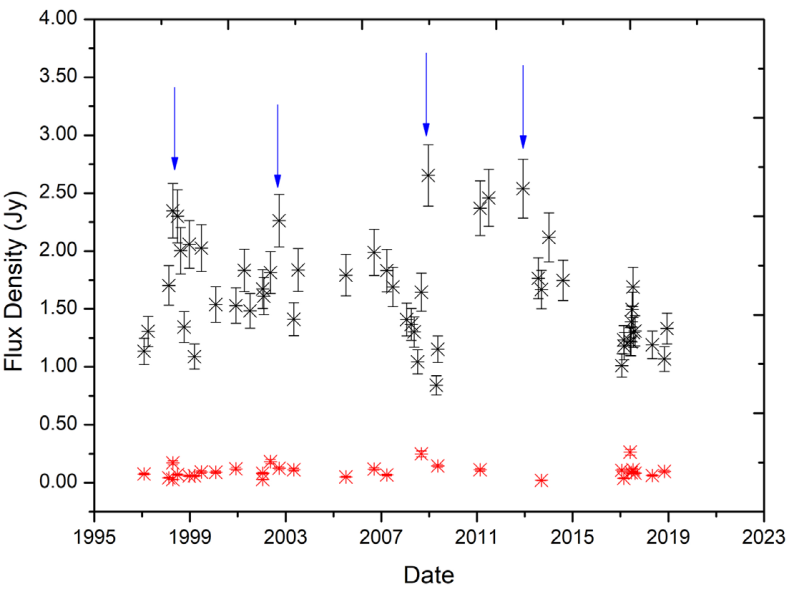

Figure 3. Long-term monitoring of variations of the core (black) and jet flux density (red) for PKS B1144-379 over the period 1997 through 2018. The blue arrows indicate the flux-density peaks that most likely correspond to the flares that occurred in 1998 April, 2002 September, 2008 December, and 2012 December. Here, we assumed that the core is always unresolved. Data were taken from the 8.6-GHz VLBI data sets of RRFID and Astrogeo.

$(0.22 \mathrm{pc}$ at points $\mathrm{G}-\mathrm{H})$ is also consistent with the decrease in the size of the scintillating component ( $\sim 6 \mu$ as for the years 2009 and 2010 ). However, the larger size of the linear emitting region throughout the core brightening phase (points E-F) is inconsistent with the decrease in the size of the scintillating component measured in 2008 (7 $\mu \mathrm{as})$. We reported that the core appears to be expanding first at the ISS scale ( $\mu$ as) and then later at the VLBI scale (mas) indicating a new jet component moving out of the jet (refer to Paper I). Due to the irregular and sporadic timing of the VLBI observations, a direct comparison of the time of emergence of the jet component for each phase of source evolution between the ISS and the VLBI scale could not be made.

Quasar jet flaring is an intrinsic source of flux density variability that occurs on time-scales ranging from days to years (Sokolov, Marscher \& McHardy 2004). BL Lac objects are known to show frequent large-amplitude flares (Stein, Odell \& Strittmatter 1976). Our analysis shows flaring events in the BL Lac object PKS B1144-379 occur every 3-4 yr. Both the single-dish (COSMIC) and the VLBI monitoring (RRFID and Astrogeo) data show that the source was likely to have undergone a significant intrinsic evolution peak in the years 1998, 2002, 2005, 2008, and 2010 (see Figs 1 and 3). Recently, a new approach for predicting flare activity based on the modality of the flux density distribution observed in Ceduna singledish observations was investigated (Said et al. 2021). One potential origin for the flares is Quasi-Periodic Oscillations (QPOs). QPOs are

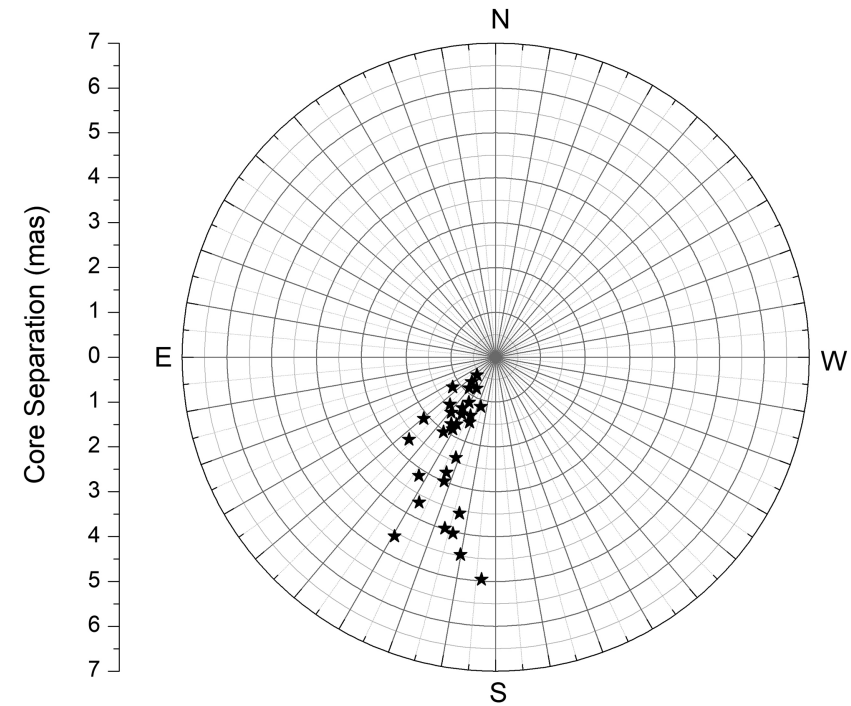

Figure 4. Variations in the jet orientation were observed for PKS B1144-379 between 1997 and 2018. The jet shows changes in the position angle, ranging from $125^{\circ}$ to $176^{\circ}$. Data were taken from the 8.6$\mathrm{GHz}$ VLBI data sets of RRFID and Astrogeo.

associated with varying accretion rates between the central black hole and the inner edge of the accretion disc (van der Klis et al. 1985). The increase in the rate of accretion will increase the amount of radiation emitted by the accretion disc. This will lead to the production of knots, which propagate outward through the jet (Lister et al. 2009b).

The origin of jet knots is poorly understood, but it is likely that they are areas of shock plasma. As the shock moves through the jet plasma at relativistic velocity, it excites electrons to very high energies, which is why they appear brighter knots in the jet. Quasar cores are not resolved at radio frequencies and knots produced by QPOs cannot be observed until they have propagated further than the core radius. The flaring event begins with the initial brightening of the core. This can be seen in Fig. 1 as points B to C, E to F, and G to $H$. Eventually, the shock plasma knot will begin to extend past the core and start to resolve as a separate emission region, marking the beginning of the jet expansion phase. The emergence of jet knots (an extended component) throughout this phase leads to an expansion of the emitting region. The core flux density will decrease while the flux density of the rest of the jet increases (points C-D). The magnetic field is assumed to dominate the jet structure. Finally, as this shock plasma knot propagates further and further down the jet, the jet increases in cross-section, the energy density in the magnetic field decreases and the particles begin to disperse. As a result, the emissivity of the synchrotron electrons also decreases. The dispersion 
of this shock plasma knot causes the flux density of the source to decrease back to its initial flux density before the flare begins (points A-B, D-E, F-G, and H-I) and reduces the linear size of the emitting region. The magnetic field is weakened and no longer dominates the structure of the jet. Our observations of PKS B1144-379 suggest that the flare that began in 2008 August (as observed in the total flux density of the COSMIC data set at point F) occurred during the jet dispersion phase. This may be due to a change in the rate of accretion between the central black hole and the inner edge of the accretion disc, or to a change in the density of the interstellar medium that caused a second flare to occur. The emergence and dispersion of jet knots is associated with the source compactness for each phase of the source evolution, which was analysed by measurements of the brightness temperature and the linear size of the emitting region. In general, the source was found to be the most compact during the core brightening phase as the flare approached. The source compactness then decreased as the jet knots began to resolve and disperse during the jet expansion and dispersion phase. As for the core brightening phase, point $\mathrm{E}$ to $\mathrm{F}$, the larger size of the linear emitting region could be caused by the blending effect of the earlier jet knots.

Our analysis shows that the jet position angle of PKS B1144-379 varies from $125^{\circ}$ to $176^{\circ}$ with an average of $150^{\circ}$ between 1997 and 2018 (refer to Fig. 4). One should note, however, the jet position angle with core separation less than 2 mas showed higher uncertainties, which could be attributed to the core-jet blending effect. We assumed that the rapid changes in the jet position angle of PKS B1144-379 are most likely due to the presence of poor visibilities in the data, which may dramatically reduce image fidelity and lead to the appearance of non-robust emission regions at an incorrect position angle. In our study, the measurement of the jet component's apparent speed could not be made due to the irregular timing and the limited number of observing epochs. Identification of the jet components is difficult to perform, as the plot of separation of these components from the core in Fig. 4 does not reveal any distinct pattern. Regular flaring events, which appear to occur every $\sim 3-4 \mathrm{yr}$, are likely to contribute to the blending effect between new and earlier emergence of jet knots. In their study of a relativistic jet analysis, Piner et al. (2007) reported that PKS B1144-379 had an apparent jet speed of $-10.6 \pm 7.7 c$. The study was conducted using 10 epochs of VLBI images from the RRFID observed between 1997 and 1999. However, due to their limited data, we have decided not to take into account the measured value. The value of this apparent jet speed is also inconsistent with data outside the period of their observation.

\section{CONCLUSIONS}

We used $9 \mathrm{yr}$ of high-cadence single-dish flux-density-monitoring data of PKS B1144-379, obtained from the COSMIC project at $6.7 \mathrm{GHz}$ to derive variability time-scale, brightness temperature, and linear size of the emitting region. Our study of the source evolution has been combined with 8.6-GHz VLBI images from RRFID and Astrogeo data sets. Over the 20-yr interval of VLBI data, the jet shows changes in position angle, ranging from $125^{\circ}$ to $176^{\circ}$ with an average of $150^{\circ}$. We assumed that the rapid changes in the jet position angle of PKS B1144-379 are most likely due to the presence of poor visibilities in the data, which may dramatically reduce image fidelity and lead to the appearance of non-robust emission regions at an incorrect position angle.

Our understanding of jet dynamics was remarkably enhanced by combining single-dish (COSMIC) and VLBI monitoring data. The Ceduna single-dish data complements the VLBI monitoring by providing a direct comparison between changes in the size of the scintillating component ( $\mu$ as-scale) and the linear size of the emitting region (parsec-scale) with the existence of the jet component in the VLBI image (mas-scale). Furthermore, prediction of flaring activity and evolution of the morphology of the parsec-scale jet were investigated comprehensively. In the future, ideally high cadence VLBI imaging with a high dynamic range at higher frequencies (8.4 GHz and above) for at least $6 \mathrm{yr}$ (approximately two cycles of flaring events) would be required to further investigate the possible cause of variations in the jet position angle.

\section{ACKNOWLEDGEMENTS}

We would like to thank Masanori Nakamura, Denise Gabuzda, and Yuri Kovalev for the helpful discussion. This research has made use of material from The Astrogeo VLBI FITS Image data base and The United States Naval Observatory (USNO) Radio Reference Frame Image Database (RRFID) projects.

\section{DATA AVAILABILITY}

The Ceduna data used in this study are available on reasonable request to the corresponding author, [N.M.M.S]. The Radio Reference Frame Image Database (RRFID) and Astrogeo data that support the findings of this study are available on request and openly available at https://www.usno.navy.mil/USNO/astrometry/vlbi-products/rrfid and http://astrogeo.org/vlbi-images/, respectively.

\section{REFERENCES}

Antonucci R. R. J., 1986, ApJ, 304, 634

Asada K., Nakamura M., 2012, ApJ, 745, L28

Blanchard J. M., 2013, PhD thesis, Univ. Tasmania

Blandford R. D., Königl A., 1979, ApJ, 232, 34

Blandford R. D., Payne D. G., 1982, MNRAS, 199, 883

Blandford R. D., Znajek R. L., 1977, MNRAS, 179, 433

Böttcher M., Baring M. G., Liang E. P., Summerlin E. J., Fu W., Smith I. A., Roustazadeh P., 2015, in Massaro F., Cheung C. C., Lopez E., Siemiginowska A., eds, Proc. IAU Symp., Vol. 313, Extragalactic Jets from Every Angle. Kluwer, Dordrecht, p. 153

Britzen S. et al., 2008, A\&A, 484, 119

Britzen S. et al., 2010, A\&A, 511, A57

Burbidge G. R., Jones T. W., Odell S. L., 1974, ApJ, 193, 43

Camenzind M., Krockenberger M., 1992, A\&A, 255, 59

Cao X., 2004, ApJ, 609, 80

Cohen M. H. et al., 2014, ApJ, 787, 151

Cohen M. H. et al., 2015, ApJ, 803, 3

Gabuzda D., 2018, Galaxies, 7, 5

Gorshkov A. G., Ipatov A. V., Ipatova I. A., Konnikova V. K., Mardyshkin V. V., Mingaliev M. G., Kharinov M. A., 2018, Astron. Rep., 62, 183

Hardee P. E., 2011, in Romero G. E., Sunyaev R. A., Belloni T., eds, Proc. IAU Symp., Vol. 275, Jets at All Scales. Kluwer, Dordrecht, p.41

Jorstad S. G., Marscher A. P., Mattox J. R., Aller M. F., Aller H. D., Wehrle A. E., Bloom S. D., 2001, ApJ, 556, 738

Karamanavis V. et al., 2016, A\&A, 586, A60

Kellermann K. I. et al., 2004, ApJ, 609, 539

Lister M. L. et al., 2013, AJ, 146, 120

Lister M. L., 2001, ApJ, 562, 208

Lister M. L., Homan D. C., Kadler M., Kellermann K. I., Kovalev Y. Y., Ros E., Savolainen T., Zensus J. A., 2009a, ApJ, 696, L22

Lister M. L., Homan D. C., Kadler M., Kellermann K. I., Kovalev Y. Y., Ros E., Savolainen T., Zensus J. A., 2009b, ApJ, 696, L22

Lobanov A. P., 1998, A\&A, 330, 79

Lovelace R. V. E., Li H., Koldoba A. V., Ustyugova G. V., Romanova M. M., 2002, ApJ, 572, 445 
Macquart J.-P., Jauncey D. L., 2002, ApJ, 572, 786

McCulloch P. M., Ellingsen S. P., Jauncey D. L., Carter S. J. B., Cimò G., Lovell J. E. J., Dodson R. G., 2005, AJ, 129, 2034

Marscher A. P., Gear W. K., 1985, ApJ, 298, 114

Marscher A., Jorstad S. G., Larionov V. M., Aller M. F., Lähteenmäki A., 2011, A\&A, 32, 233

Nakamura M., Asada K., 2013, ApJ, 775, 118

Nakamura M., Meier D. L., 2004, ApJ, 617, 123

Nakamura M., Meier D. L., 2014, ApJ, 785, 152

Nakamura M., Garofalo D., Meier D. L., 2010, ApJ, 721, 1783

Ojha R. et al., 2010, A\&A, 519, A45

Padovani P., Giommi P., 1995, MNRAS, 277, 1477

Piner B. G. et al., 2012, ApJ, 758, 84

Piner B. G., Mahmud M., Fey A. L., Gospodinova K., 2007, AJ, 133, 2357

Plavin A. V., Kovalev Y. Y., Pushkarev A. B., Lobanov A. P., 2019, MNRAS, 485,1822

Polatidis A. G., Wilkinson P. N., Xu W., Readhead A. C. S., Pearson T. J., Taylor G. B., Vermeulen R. C., 1995, ApJS, 98, 1

Said N. M. M., Ellingsen S. P., Bignall H. E., Shabala S., McCallum J. N., Reynolds C., 2020, MNRAS, 498, 4615 (Paper I)

Said N. M. M., Ellingsen S. P., Liu J., McCallum J. N., Bignall H. E., Shabala S., Reynolds C., 2021, MNRAS, 506, 288
Shabala S., Titov O., Lovell J., McCallum J., Blanchard J., Watson C., Dickey J., 2012a, in Baver D., Behrend K. D., eds, Seventh General Meeting (GM2012) of the international VLBI Service for Geodesy and Astrometry (IVS). National Aeronautics and Space Administration, Madrid, Spain, p. 329

Shabala S. S., Santoso J. S., Godfrey L. E. H., 2012b, ApJ, 756, 161

Shepherd M. C., 1997, in Hunt G., Payne H., eds, ASP Conf. Ser.Vol.125, Astronomical Data Analysis Software and Systems VI. Astron. Soc. Pac., San Francisco, p.77

Sokolov A., Marscher A. P., McHardy I. M., 2004, ApJ, 613, 725

Stein W. A., Odell S. L., Strittmatter P. A., 1976, ARA\&A, 14, 173

Tateyama C. E., Kingham K. A., Kaufmann P., Piner B. G., de Lucena A. M. P., Botti L. C. L., 1998, ApJ, 500, 810

Tchekhovskoy A., Bromberg O., 2016, MNRAS, 461, L46

Tchekhovskoy A., Narayan R., McKinney J. C., 2011, MNRAS, 418, L79

Terasranta H., Valtaoja E., 1994, A\&A, 283, 51

Urry C. M., Padovani P., 1995, Publ. Astron. Soc. Pac., 107, 803

van der Klis M., Jansen F., van Paradijs J., Lewin W. H. G., van den Heuvel E. P. J., Trumper J. E., Szatjno M., 1985, Nature, 316, 225

Zensus J. A., Ros E., Kellermann K. I., Cohen M. H., Vermeulen R. C., Kadler M., 2002, AJ, 124, 662

\section{APPENDIX A: MODELFit RESULTS OF THE VLBI IMAGING AT 8.6 GHZ FOR PKS B1144-379}

Table A1. MODELFIT results of the VLBI imaging at $8.6 \mathrm{GHz}$ for PKS B1144-379. Columns from left to right side: (1) observing epoch, (2) RMS flux density in the map, (3) and (4) integrated core flux density and its uncertainty, (5) and (6) integrated jet flux density and its uncertainty, (7) and (8) radial separation from the core and its uncertainty, and (9) and (10) jet position angle with respect to the core and its uncertainty.

\begin{tabular}{|c|c|c|c|c|c|c|c|c|c|}
\hline Date & rms $\left(\mathrm{Jy} \mathrm{beam}^{-1}\right)$ & $S_{\text {core }}(\mathrm{Jy})$ & $\sigma(\mathrm{Jy})$ & $S_{\text {jet }}(\mathrm{Jy})$ & $\sigma(\mathrm{Jy})$ & Radius (mas) & $\sigma(\mathrm{mas})$ & $\mathrm{PA}\left({ }^{\circ}\right)$ & $\sigma\left({ }^{\circ}\right)$ \\
\hline 31/01/1997 & 0.0054 & 1.13 & 0.113 & 0.08 & 0.008 & 3.00 & 0.70 & 157 & 13 \\
\hline $1 / 04 / 1997^{\mathrm{a}}$ & 0.0063 & 1.31 & 0.131 & & & & & & \\
\hline $10 / 02 / 1998$ & 0.0018 & 1.70 & 0.170 & 0.04 & 0.004 & 3.57 & 1.00 & 167 & 16 \\
\hline \multirow[t]{2}{*}{$16 / 04 / 1998^{b}$} & 0.0025 & 2.35 & 0.235 & 0.17 & 0.017 & 0.77 & 0.51 & 136 & 34 \\
\hline & & & & 0.03 & 0.003 & 2.67 & 0.54 & 134 & 11 \\
\hline $25 / 06 / 1998$ & 0.0029 & 2.30 & 0.230 & 0.07 & 0.007 & 4.04 & 0.51 & 166 & 7 \\
\hline $10 / 08 / 1998^{a}$ & 0.0028 & 2.00 & 0.200 & & & & & & \\
\hline $1 / 10 / 1998^{a}$ & 0.0051 & 1.34 & 0.134 & & & & & & \\
\hline $22 / 12 / 1998$ & 0.0045 & 2.06 & 0.206 & 0.06 & 0.006 & 4.47 & 0.44 & 170 & 6 \\
\hline 9/03/1999 & 0.0028 & 1.09 & 0.109 & 0.06 & 0.006 & 4.96 & 0.45 & 176 & 5 \\
\hline 22/06/1999 & 0.0016 & 2.03 & 0.203 & 0.09 & 0.009 & 1.58 & 0.97 & 141 & 32 \\
\hline $31 / 01 / 2000$ & 0.0013 & 1.54 & 0.154 & 0.09 & 0.009 & 1.74 & 0.88 & 150 & 27 \\
\hline $4 / 12 / 2000$ & 0.0021 & 1.53 & 0.153 & 0.12 & 0.012 & 1.17 & 0.43 & 149 & 20 \\
\hline $9 / 04 / 2001^{\mathrm{a}}$ & 0.0034 & 1.83 & 0.183 & & & & & & \\
\hline $5 / 07 / 2001^{\mathrm{a}}$ & 0.0037 & 1.48 & 0.148 & & & & & & \\
\hline \multirow[t]{2}{*}{$17 / 01 / 2002^{b}$} & 0.0018 & 1.67 & 0.167 & 0.08 & 0.008 & 1.47 & 0.95 & 136 & 33 \\
\hline & & & & 0.03 & 0.003 & 3.98 & 2.22 & 164 & 29 \\
\hline $1 / 02 / 2002^{a}$ & 0.0051 & 1.61 & 0.161 & & & & & & \\
\hline $15 / 05 / 2002$ & 0.0031 & 1.82 & 0.182 & 0.18 & 0.018 & 2.41 & 0.37 & 158 & 9 \\
\hline 25/09/2002 & 0.0018 & 2.26 & 0.226 & 0.12 & 0.012 & 1.87 & 0.55 & 149 & 16 \\
\hline $7 / 05 / 2003$ & 0.0018 & 1.41 & 0.141 & 0.11 & 0.011 & 1.35 & 0.92 & 147 & 34 \\
\hline $9 / 07 / 2003^{a}$ & 0.0027 & 1.84 & 0.184 & & & & & & \\
\hline $8 / 07 / 2005$ & 0.0026 & 1.79 & 0.179 & 0.05 & 0.005 & 3.15 & 0.35 & 147 & 6 \\
\hline $13 / 09 / 2006$ & 0.0039 & 1.99 & 0.199 & 0.12 & 0.012 & 2.11 & 0.16 & 131 & 4 \\
\hline $28 / 03 / 2007$ & 0.0032 & 1.83 & 0.183 & 0.07 & 0.007 & 1.17 & 0.96 & 125 & 39 \\
\hline $26 / 06 / 2007^{a}$ & 0.0033 & 1.69 & 0.169 & & & & & & \\
\hline $23 / 01 / 2008^{a}$ & 0.0032 & 1.41 & 0.141 & & & & & & \\
\hline $3 / 04 / 2008^{a}$ & 0.0024 & 1.37 & 0.137 & & & & & & \\
\hline $15 / 05 / 2008^{a}$ & 0.0040 & 1.30 & 0.130 & & & & & & \\
\hline $9 / 07 / 2008^{a}$ & 0.0027 & 1.04 & 0.104 & & & & & & \\
\hline
\end{tabular}


Table A1 - continued

\begin{tabular}{|c|c|c|c|c|c|c|c|c|c|}
\hline Date & rms $\left(\mathrm{Jy} \mathrm{beam}^{-1}\right)$ & $S_{\text {core }}(\mathrm{Jy})$ & $\sigma(\mathrm{Jy})$ & $S_{\text {jet }}(\mathrm{Jy})$ & $\sigma(\mathrm{Jy})$ & Radius (mas) & $\sigma$ (mas) & $\mathrm{PA}\left({ }^{\circ}\right)$ & $\sigma\left({ }^{\circ}\right)$ \\
\hline $3 / 09 / 2008$ & 0.0032 & 1.65 & 0.165 & 0.25 & 0.025 & 0.57 & 0.36 & 134 & 32 \\
\hline $18 / 12 / 2008^{a}$ & 0.1736 & 2.65 & 0.265 & & & & & & \\
\hline $22 / 04 / 2009^{a}$ & 0.0019 & 0.84 & 0.084 & & & & & & \\
\hline $14 / 05 / 2009$ & 0.0028 & 1.15 & 0.115 & 0.15 & 0.015 & 0.82 & 0.21 & 149 & 14 \\
\hline $16 / 02 / 2011$ & 0.0010 & 2.37 & 0.237 & 0.11 & 0.011 & 1.16 & 0.49 & 163 & 23 \\
\hline $28 / 06 / 2011^{\mathrm{a}}$ & 0.0020 & 2.46 & 0.246 & & & & & & \\
\hline $6 / 12 / 2012^{\mathrm{a}}$ & 0.0029 & 2.54 & 0.254 & & & & & & \\
\hline $24 / 07 / 2013^{a}$ & 0.0038 & 1.77 & 0.177 & & & & & & \\
\hline $11 / 09 / 2013$ & 0.0031 & 1.67 & 0.167 & 0.02 & 0.002 & 1.42 & 0.60 & 157 & 23 \\
\hline $4 / 01 / 2014^{\mathrm{a}}$ & 0.0026 & 2.12 & 0.212 & & & & & & \\
\hline $9 / 08 / 2014^{\mathrm{a}}$ & 0.0027 & 1.75 & 0.175 & & & & & & \\
\hline $22 / 01 / 2017$ & 0.0014 & 1.01 & 0.101 & 0.11 & 0.011 & 1.48 & 1.13 & 149 & 37 \\
\hline 20/02/2017 & 0.0011 & 1.23 & 0.123 & 0.04 & 0.004 & 1.56 & 0.75 & 158 & 26 \\
\hline $25 / 02 / 2017^{a}$ & 0.0016 & 1.18 & 0.118 & & & & & & \\
\hline 28/05/2017 & 0.0018 & 1.22 & 0.122 & 0.26 & 0.026 & 0.91 & 0.70 & 139 & 38 \\
\hline $11 / 06 / 2017^{a}$ & 0.0018 & 1.22 & 0.122 & & & & & & \\
\hline 16/06/2017 & 0.0016 & 1.39 & 0.139 & 0.09 & 0.009 & 3.66 & 0.46 & 152 & 7 \\
\hline $28 / 06 / 2017^{a}$ & 0.0034 & 1.50 & 0.150 & & & & & & \\
\hline $9 / 07 / 2017$ & 0.0025 & 1.69 & 0.169 & 0.11 & 0.011 & 2.80 & 0.45 & 157 & 9 \\
\hline $16 / 07 / 2017^{a}$ & 0.0018 & 1.30 & 0.130 & & & & & & \\
\hline $12 / 08 / 2017$ & 0.0019 & 1.31 & 0.131 & 0.08 & 0.008 & 4.59 & 0.51 & 151 & 6 \\
\hline $30 / 04 / 2018$ & 0.0009 & 1.19 & 0.119 & 0.06 & 0.006 & 2.03 & 0.69 & 145 & 19 \\
\hline $3 / 11 / 2018$ & 0.0019 & 1.07 & 0.107 & 0.10 & 0.010 & 1.79 & 0.39 & 146 & 12 \\
\hline $14 / 12 / 2018^{a}$ & 0.0011 & 1.33 & 0.133 & & & & & & \\
\hline
\end{tabular}

${ }^{a}$ Compact. ${ }^{b}$ More than 1 jet/extended component.

APPENDIX B: VLBI IMAGING AT 8.6 GHZ FOR PKS B1144-379 

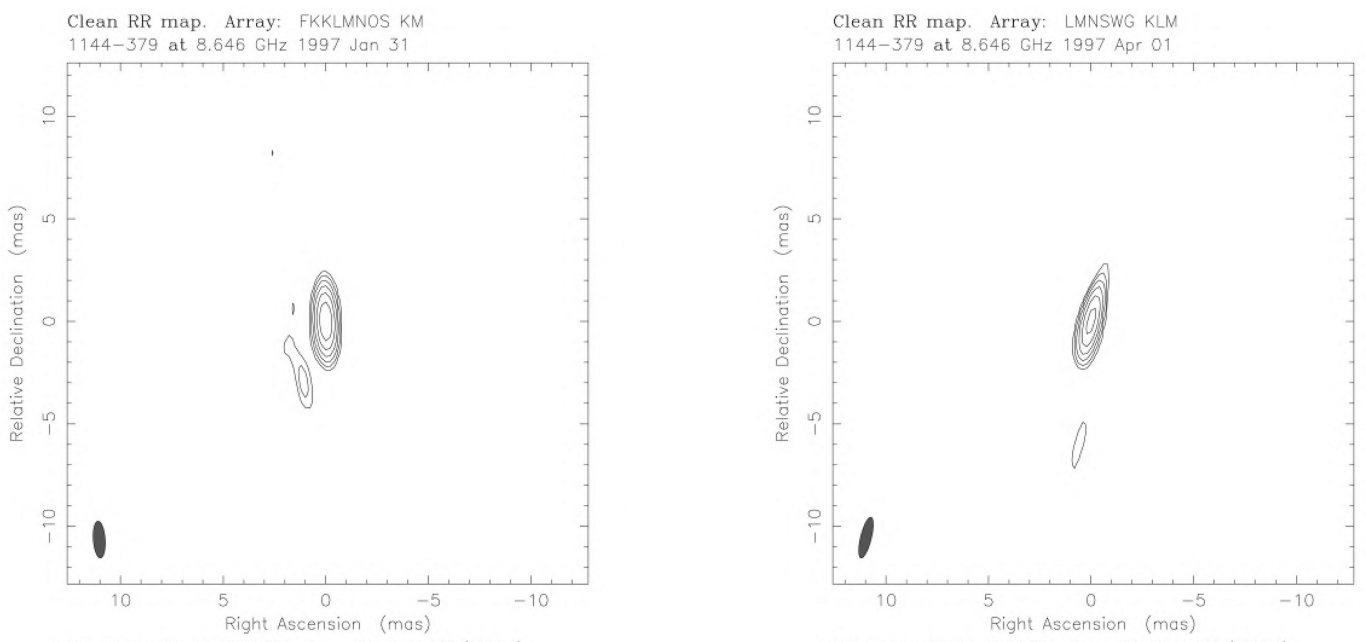

Map center: RA: 1147 01.371, Dec: -381211.023 (2000.0 Map peak: $0.907 \mathrm{Jy} /$ beam

Contours: 0.907 Jy

Clean RR map. Array: FKKLMNOPSG FKKLMOP

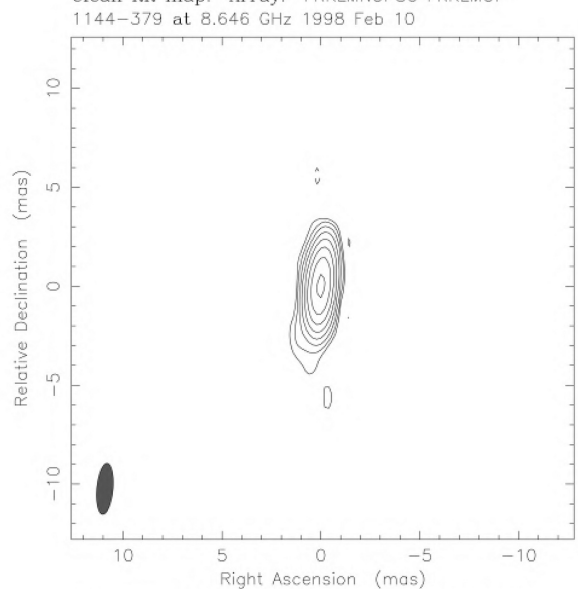

Right Ascension (mas)

Map center: RA: 114701.371 . Dec: $-381211.023(2000.0)$

Map peak: $1.56 \mathrm{Jy} / \mathrm{beam}$

Contours: $0.0106 \mathrm{Jy} /$ beam $x\left(\begin{array}{lllllll}-1 & 1 & 2 & 48 & 8 & 16 & 3264\end{array}\right.$

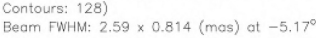

Clean RR map. Array: FGKKLNOPS

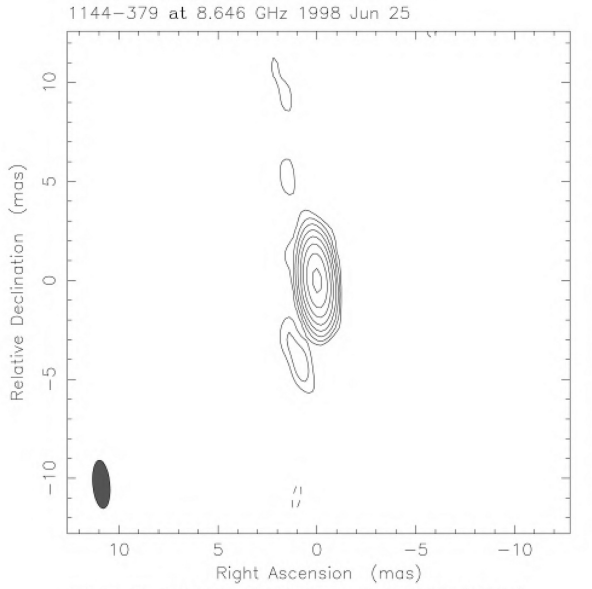

Map center: RA: 1147 01.371. Dec: -381211.023 (2000.0)

Map peak: $2.2 \mathrm{Jy} / \mathrm{beom}$

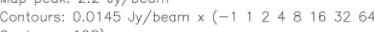

Beam FWHM: $2.45 \times 0.866$ (mas) at $5.11^{\circ}$

Map center: RA: 114701.371 , Dec: -381211.023 (2000.0) Map peak: $1.13 \mathrm{Jy} / \mathrm{beam}$

Bearn FWHM: $2.03 \times 0.527$ (mos) 0 . $\left.-139^{\circ}\right)$

Clean RR map. Array: FKKLMOP FGKKLMNOPSW

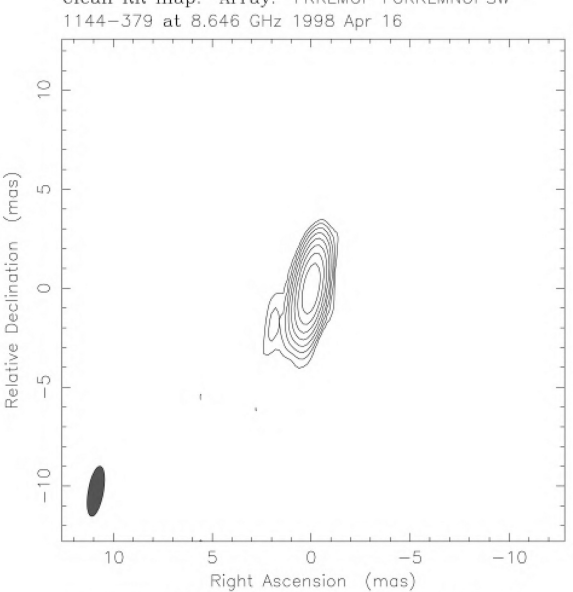

Map center: RA: 1147 01.371, Dec: $-381211.023(2000.0)$

Map peak: 2.36 Jy/beam

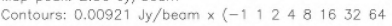

Contours: 128)

Clean RR map. Array: KM FKLNOPSKMG

$1144-379$ at $8.646 \mathrm{GHz} 1998$ Aug 10

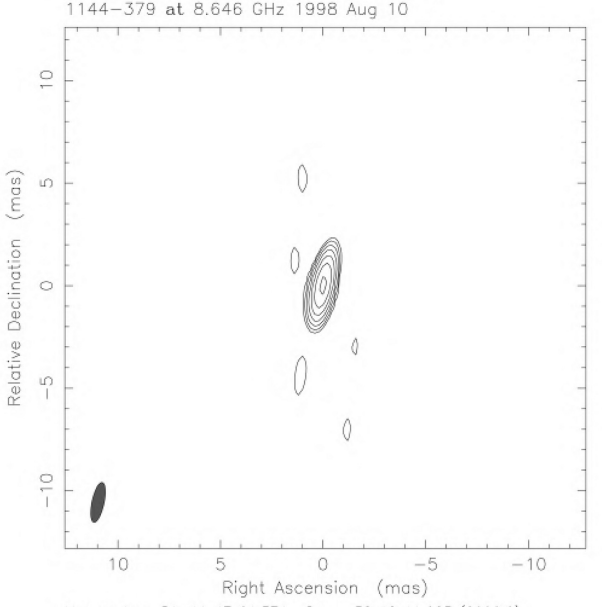

Map center: RA: 1147 01.371, Dec: $-381211.023(2000.0)$

Map peak: 1.88 Jy/bearn

Contours: 0.0247 Jy/beam $\times(12248163264)$

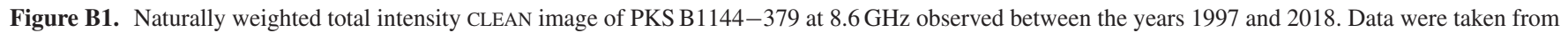
the RRFID and Astrogeo VLBI FITS image data base. 
Evolution of PKS B1144-379

2889

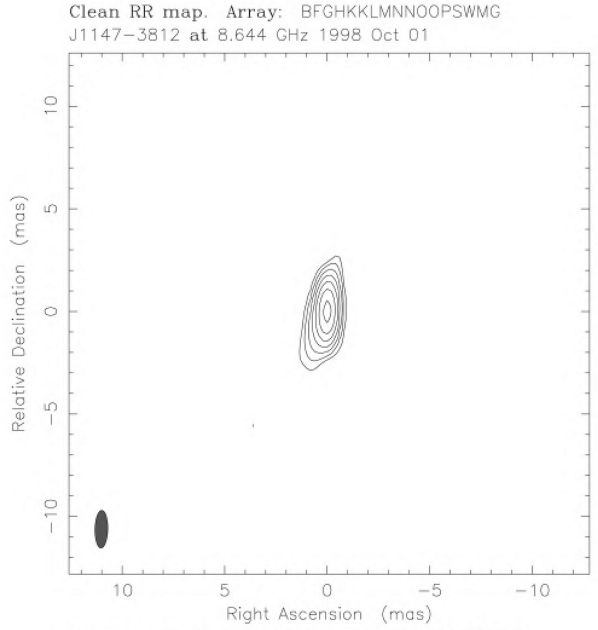

Mop center: RA: 1147 01.371. Dec: -381211.024 (2000.0) Map peak: $1.5 \mathrm{Jy} /$ beam 15.83264 Clean RR map. Array: BFGGHKLNOPSWGHKMMNOW

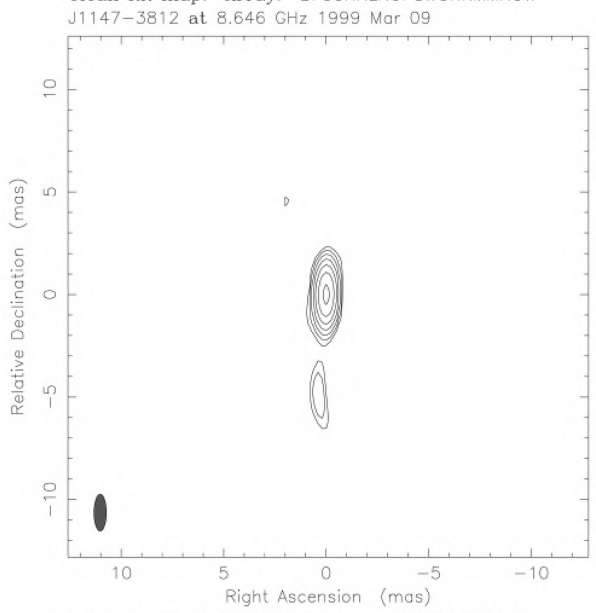

Map center: RA: 1147 01.371. Dec: -38 1211.024 (2000.0) peak: $0.917 \mathrm{Jy} / \mathrm{beam}$ Beam FWHM: $1.8 \times 0.607$ (mo

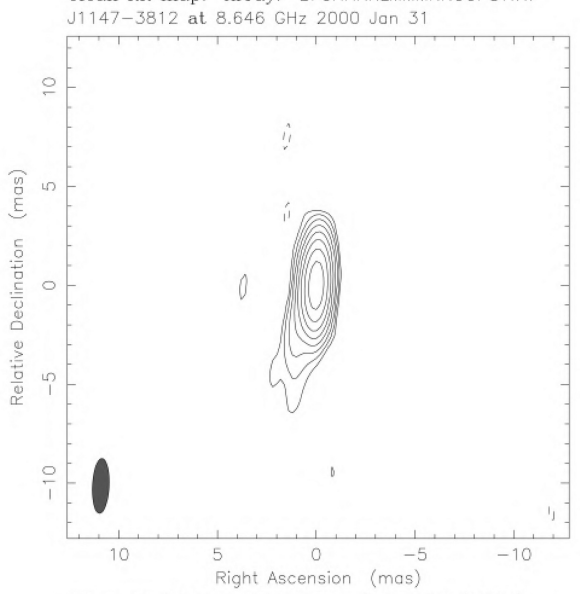

Map center: RA: 1147 01.371. Dec: $-381211.024(2000.0)$ Mp peak: $1.52 \mathrm{Jy} /$ beam Contours: $0.00694 \mathrm{Jy} /$ beam $x<(-1112.48163264$ Contours: 128

Beam FWHM: $2.78 \times 0.84$ (mas) at $-2.76^{\circ}$

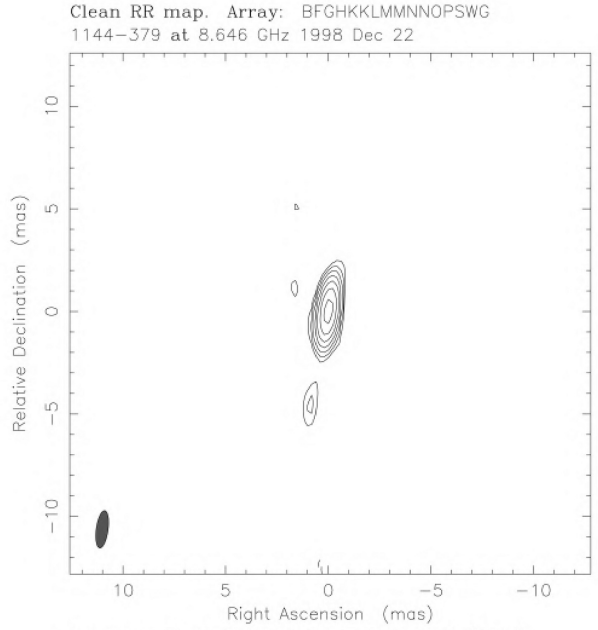

Map center: RA: 1147 01.371. Dec: -381211.023 (2000.0) peak: $1.86 \mathrm{Jy} /$ beam

(1.84 $\times$ boom $\times(-1,124.8163264$

Array: BEGHHKKLMMNNOOPSTWM

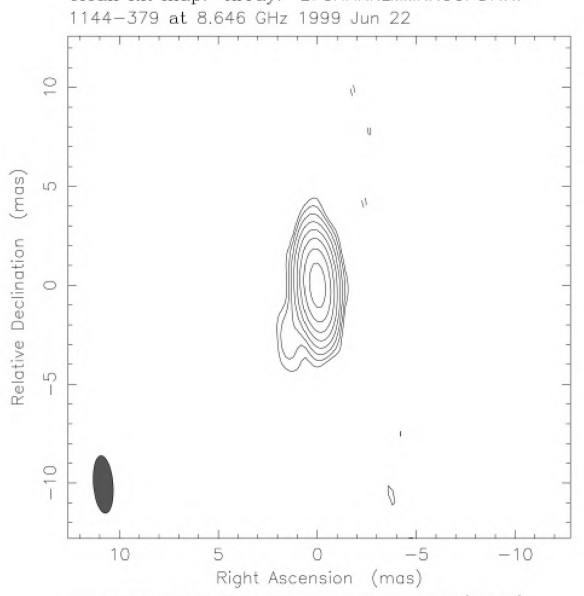

Map center: RA: 1147 01.371, Dec: -381211.023 (2000.0) Jy/beam $x(-1,1248163264$ Beam FWHM: $2.93 \times 0.994$ (mos) at $4.73^{\circ}$

Clean RR map. Array: BFGGHHKKLMMMNNOPSTWw J1147-3812 at $8.646 \mathrm{GHz} 2000$ Dec 04

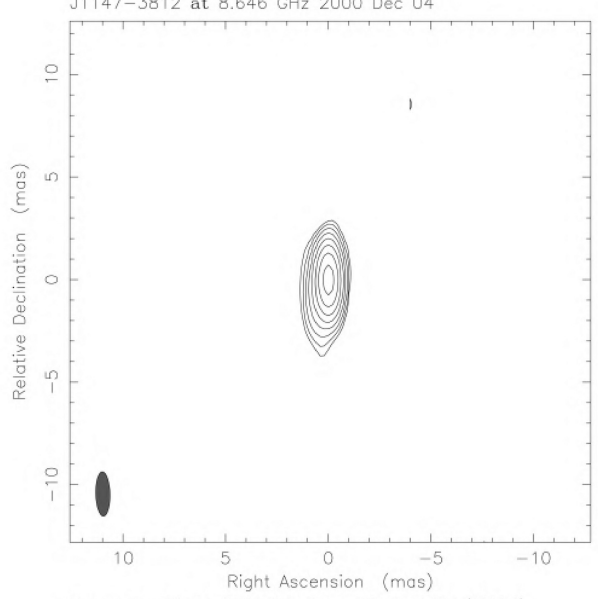

Map center: RA: 1147 01.371. Dec: -381211.024 (2000.0) Map peak: $1.38 \mathrm{Jy} /$ beam

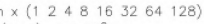

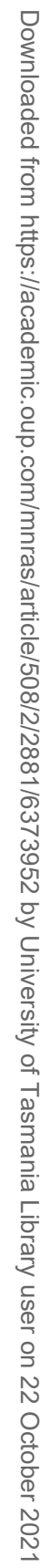

Figure B1 - continued

MNRAS 508, 2881-2896 (2021) 

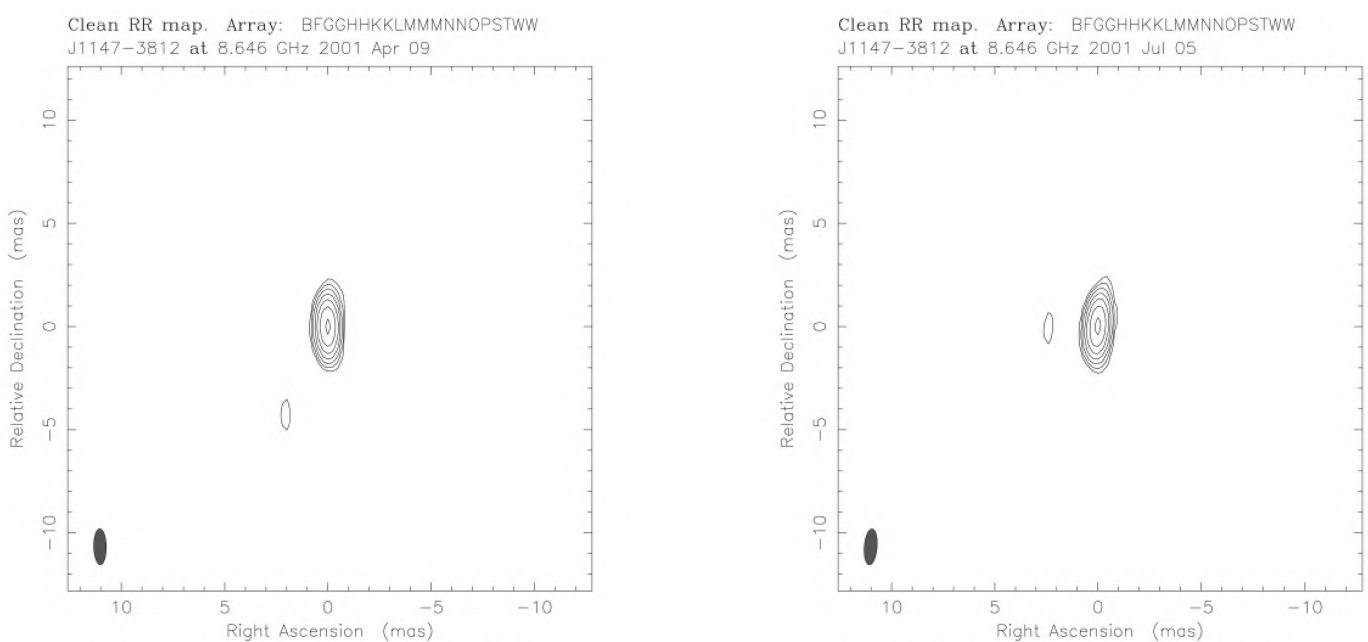

Map center: RA: 1147 01.371, Dec: -381211.024 (2000.0) Map peak: $1.01 \mathrm{t}$ J

(1) $0223 \mathrm{Jy} / \mathrm{heam} \times(1248153264)$

Beam FWHM: $1.73 \times 0.603$ (mas) of $0.964^{\circ}$

Clean RR map. Array: BFGGHKLMMNNOOPSTWKW $\mathrm{J} 1147-3812$ at $8.646 \mathrm{GHz} 2002$ Jan 17

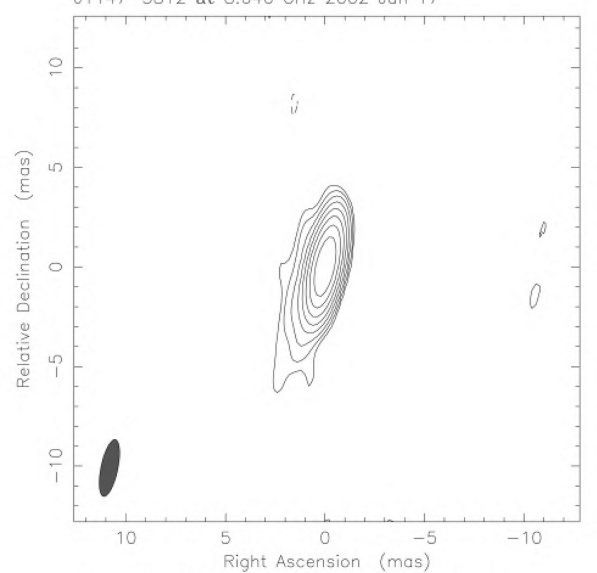

Right Ascension (mas)

Map center: RA: 1147 01.371. Dec: $-381211.024(2000.0)$

Contours: $0.00594 \mathrm{Jy} / \mathrm{beam} \times\langle-11248 \quad 163264$

Contours: 128

0.85 (mas) at $-11.8^{\circ}$

Clean RR map. Array: BFHKLNOPSM

$1147-3812$ at $8.646 \mathrm{GHz} 2002 \mathrm{May} 15$

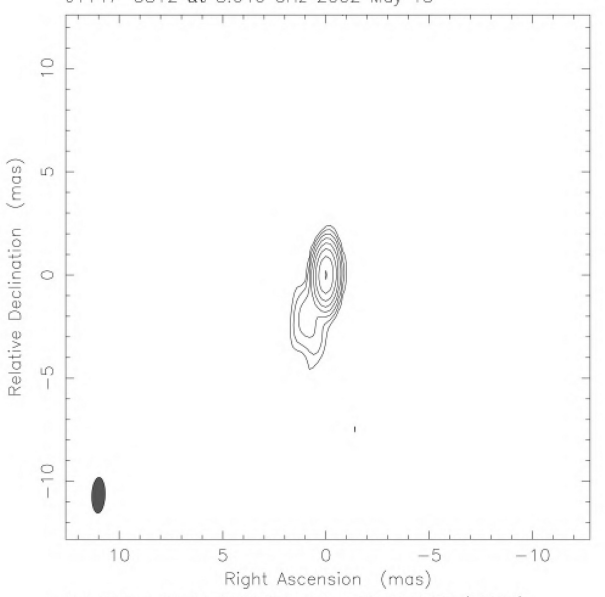

Right Ascension (mas)

Map center: RA: 1147 01.371. Dec: -38 1211.024 (2000.0)

Contours: $0.0127 \mathrm{Jy} /$ beam $\times\left(\begin{array}{llllll}1 & 2 & 4 & 8 & 16 & 32 \\ 64 & 128\end{array}\right)$

Beam FWHM: $1.73 \times 0.642$ (mas) at $-2.18^{\circ}$

Right Ascension (mas)
114701.371 . Dec: -381211.024 (2000.0)

Map center: RA: 1147 01,371

Map peak: $1.27 \mathrm{Jy} / \mathrm{feom} \times(-1) 248163264)$

Beam FWHM: $1.73 \times 0.617$ (mas) at -4.53

Clean RR map. Array: BFHKLIMNOPS

J1147-3812 at $8.646 \mathrm{GHz} 2002 \mathrm{Feb} 01$

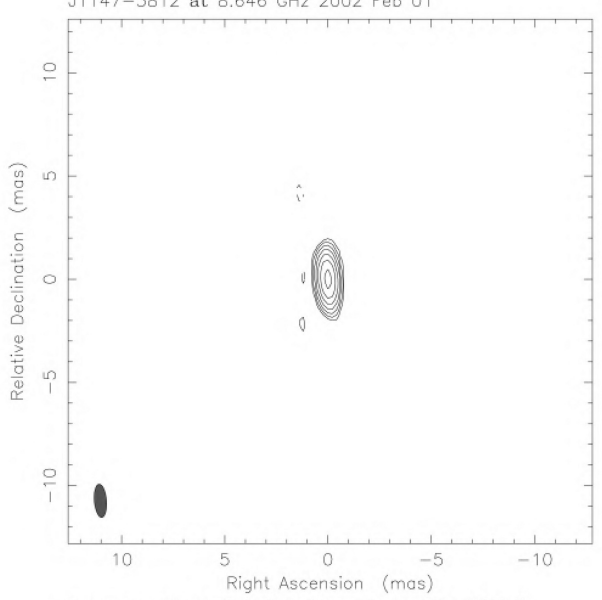

Map center: RA: 114701.371 , Dec: $-381211.024(2000.0)$

Map peak: $1.58 \mathrm{Jy} /$ beam

Contours: $0.0194 \mathrm{Jy} /$ beam x ( $-1 \quad 12248163264)$

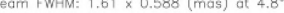

Clean RR map. Array: BFGHKKLMMMNOOPTTWW

$\mathrm{J} 1147-3812$ at $8.646 \mathrm{GHz} 2002 \mathrm{Sep} 25$

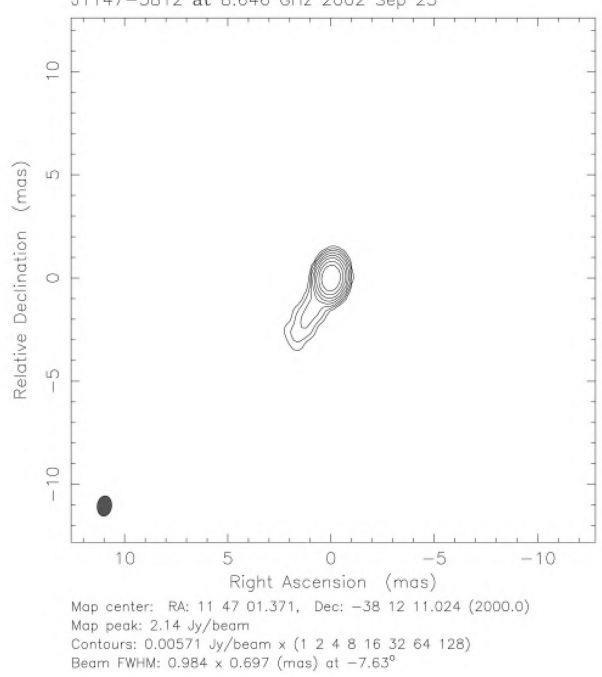

Figure B1 - continued 
Clean RR map. Array: BFGHKKLMMMNOOPSTTWW

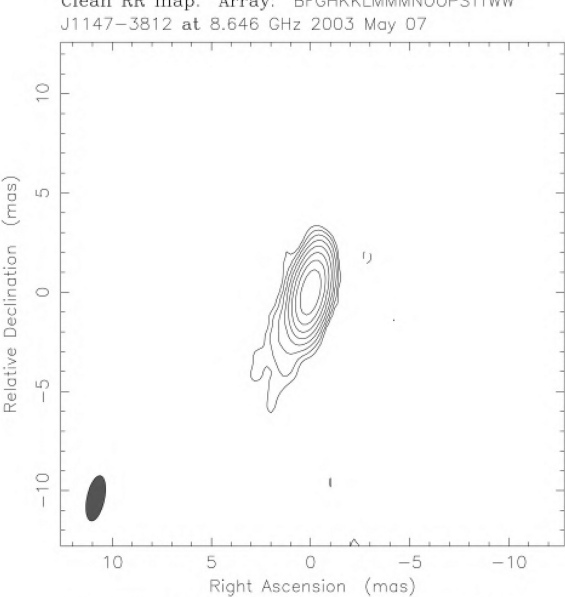

Map center: RA: 1147 01.371, Dec: -381211.024 (2000.0)

Map peak: $1.35 \mathrm{Jy} /$ bear

$\times(-11248163264$

Beam FWHM: $2.33 \times 0.886$ (mos) at -11.9

Clean RR map. Array: BFHKLMNOPS J1147-3812 at $8.646 \mathrm{GHz} 2005$ Jul 08

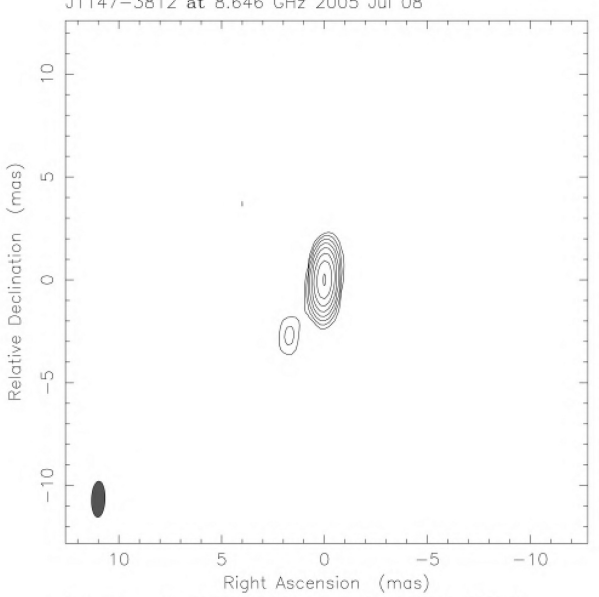

Map center: RA: 114701.3

Contours: $0.0126 \mathrm{Jy} / \mathrm{beam} \times(1,2481032$ o4 128$)$

Clean RR map. Array: VLBA Correlotor

J1147-3812 at 8.642 OHz 2007 wa

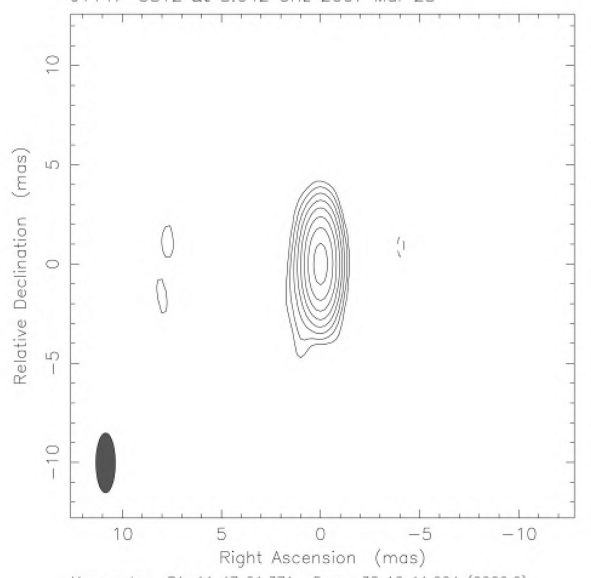

Map center: RA: 1147 01.371, Dec: $-381211.024(2000,0)$

Mop peak: $1.78 \mathrm{Jy} / \mathrm{beam}$.

Beom FWHM: $3.01 \times 0.979(\mathrm{mos})$ ot $0.0032^{\circ}$

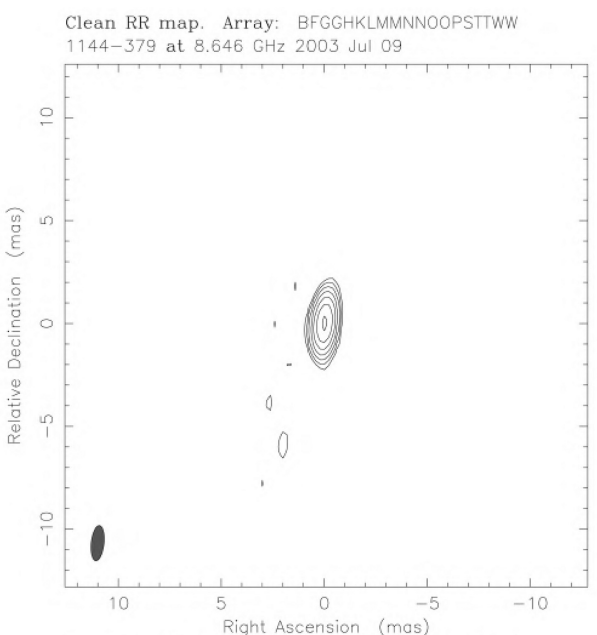

Map center: RA: 1147 01.371, Dec: $-381211.024(2000.0)$

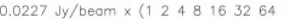

Clean RR map. Array: BFHHKLMNNOPMSSTWW $1144-379$ at $8.646 \mathrm{GHz} 2006 \mathrm{Sep} 13$

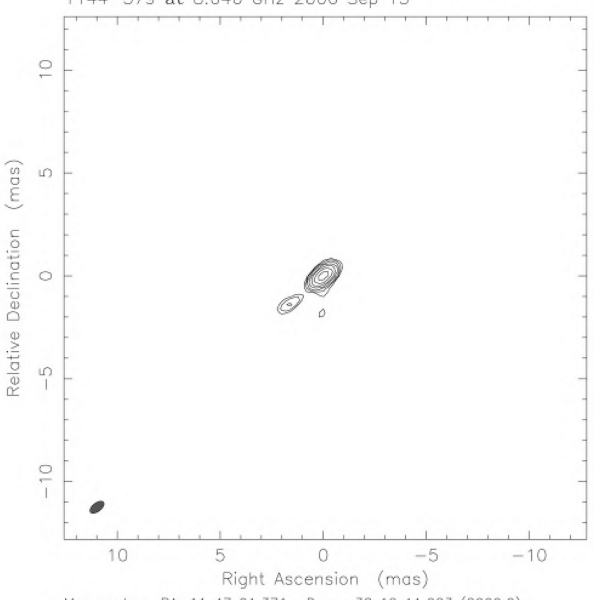

Map center: RA: 114701.3

Beam FWHM: $0.795 \times 0.412$ (mas) at $-52.8^{\circ}$

Clean RR map. Array: BFHHKKKLMNNOPSW

$1144-379$ at $8.646 \mathrm{GHz} 2007$ Jun 26

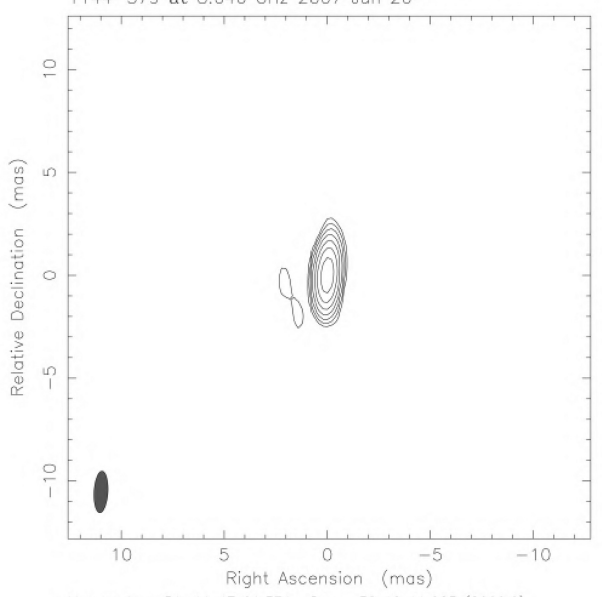

Map center: RA: 1147 01.371. Dec: -381211.023 (2000.0) Map peak: $1.55 \mathrm{Jy} / \mathrm{beam}$

Contours: 0.0145 Jy)

Figure B1 - continued 

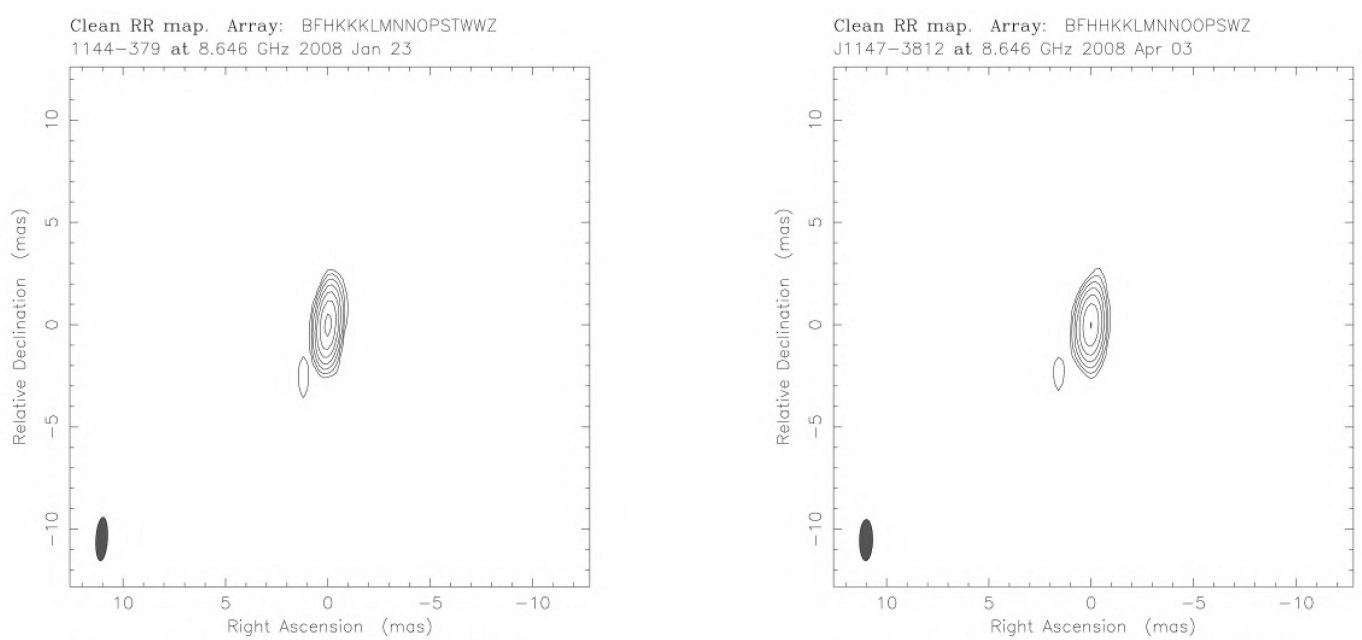

Map center: RA: 1147 01.371, Dec: -38 1211.023 (2000.0) Map peak: $1.19 \mathrm{Jy} /$ beam

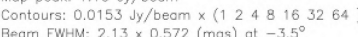

(3) $\times 0.572$ (mas) at -3.50

Clean RR map. Array: BFHKKKLMNNOPSWZ

$1144-379$ at $8.646 \mathrm{GHz} 2008$ May 15

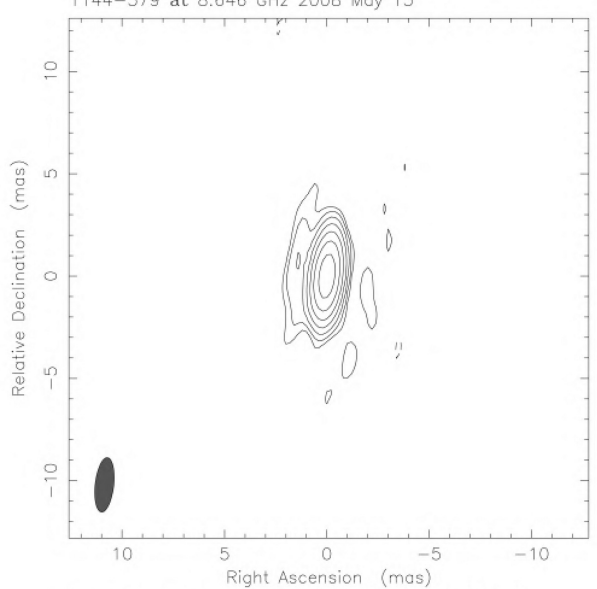

Right Ascension (mos)

Map center: RA: 114701.371 . Dec: -381211.023 (2000.0) Map peak. 1.16 Jy/beam

$(-11248163264$

Clean RR map. Array: BFHHKKLMNNOPSTWZ $1144-379$ at $8.646 \mathrm{GHz} 2008 \mathrm{Sep} 03$

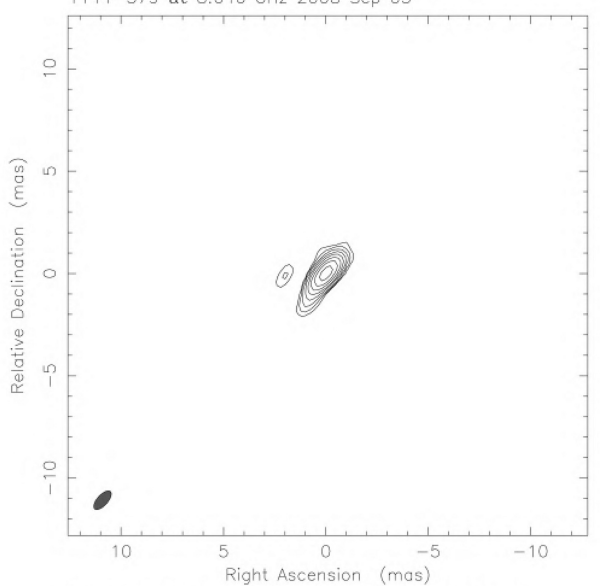

Map center: RA: 1147 01 371, Dec: -38 1211.023 (2000.0)

Map peak: $1.61 \mathrm{Jy} /$ bean

(1) 248163264128$)$

Beam FWHM: $1.14 \times 0.508$ (mas) ot $-42.4^{\circ}$

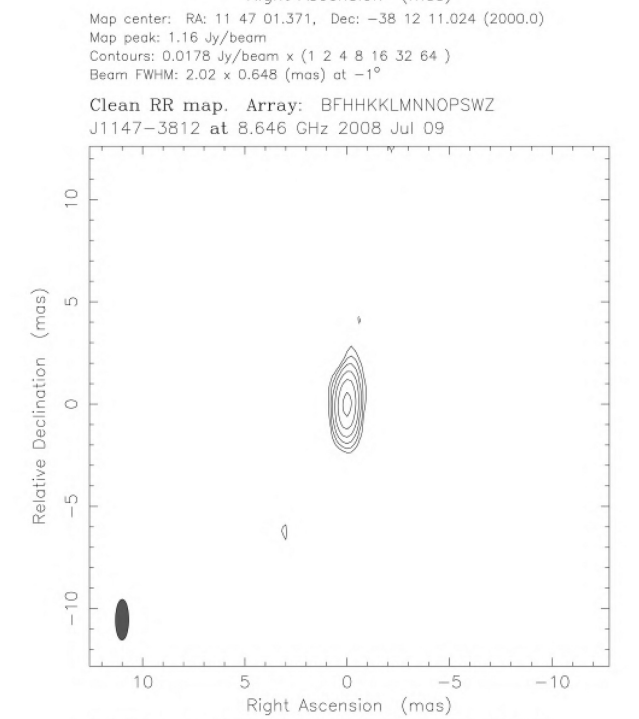

Map center: RA: 1147 01.371, Dec: -381211.024 (2000.0) Map peak: $0.839 \mathrm{Jy} / \mathrm{beam}$

Contours: $0.0212 \mathrm{Jy} / 0.0 \mathrm{~m} \times(-1) 12481632)$

Clean RR map. Array: VLBA Correlator J1147-3812 at 8.642 GHz 2008 Dec 18

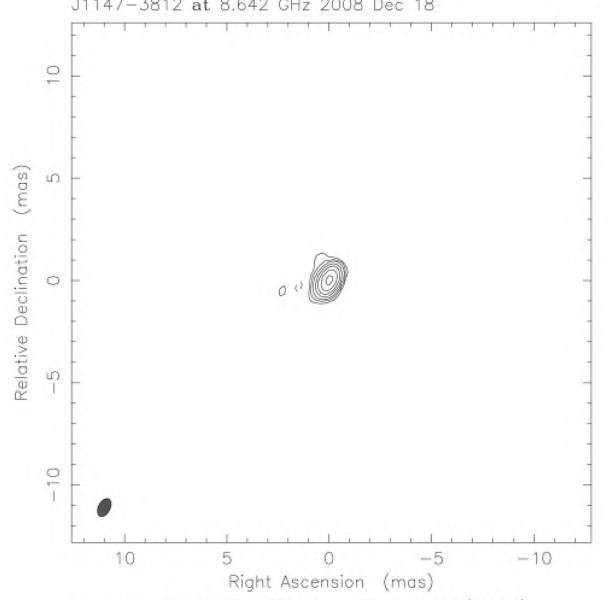

Map center: RA: 1147 01.371. Dec: -38 1211.024 (2000.0)

Map peak: $2.42 \mathrm{Jy} / \mathrm{beam}$

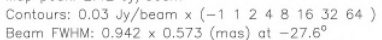

Figure B1 - continued 
Evolution of PKS B1144-379

2893

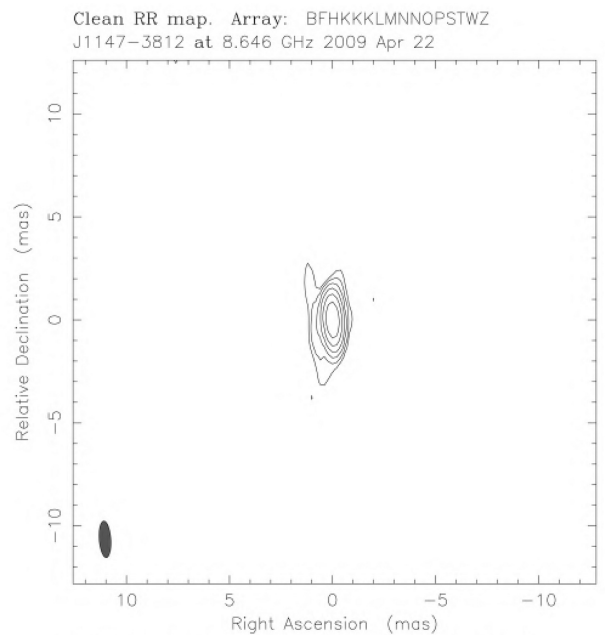

Map center: RA: 1147 01.371. Dec: -381211.024 (2000.0)

Map peak: $0.742 \mathrm{Jy} /$ bear

Contours: $0.0119 \mathrm{Jy} /$ beam $\times\left(\begin{array}{lllllll}-1 & 1 & 2 & 4 & 8 & 16 & 32\end{array}\right)$

Clean RR map. Array: VLBA Correlator

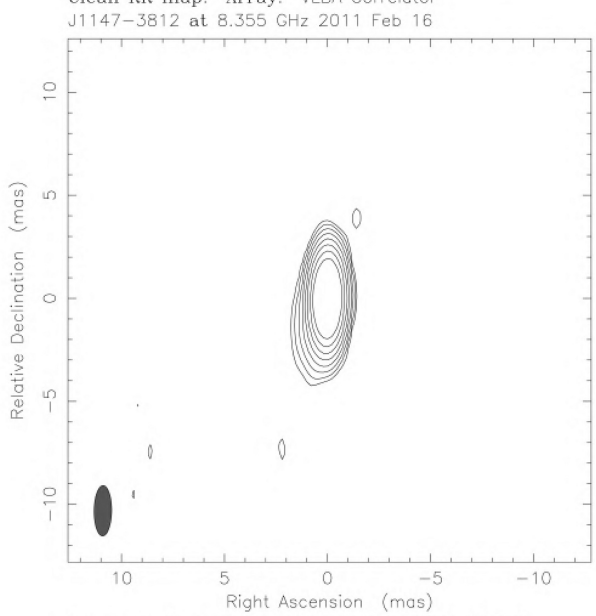

Map center: RA: 1147 01.371. Dec: -381211.024 (2000.0) Map peak: $2.32 \mathrm{Jy} /$ beam

Clean RR map. Array: VLBA Correlator

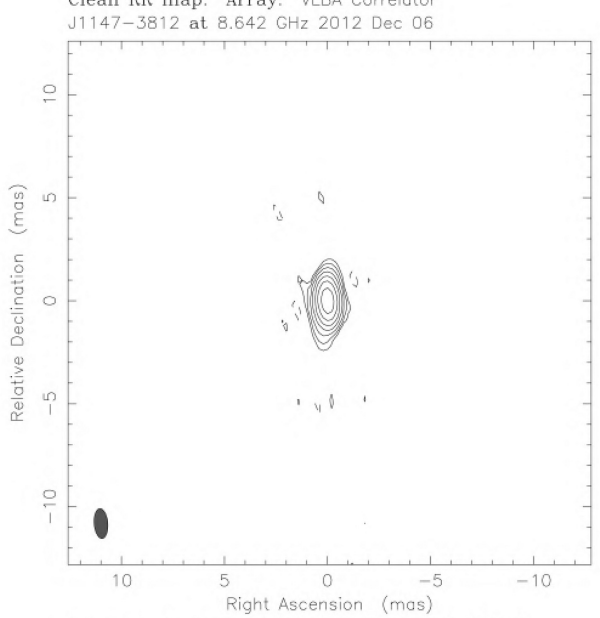

Map center: RA: 1147 01.371. Dec: $-381211.024(2000.0)$

Map peak: $2.44 \mathrm{Jy} /$ beam

.
Clean RR map. Array: BFHHKKLMNNOPSTWK

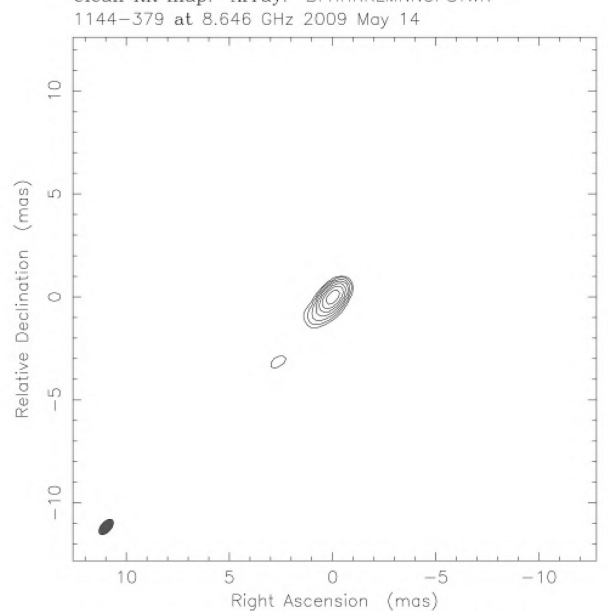

Map center: RA: 1147 01.371, Dec: -38 1211.023 (2000.0)

Map peak: $1.07 \mathrm{Jy} /$ beam

Contours: $0.00948 \mathrm{Jy} / \mathrm{beam} \times\left(\begin{array}{llllll}1 & 2 & 4 & 8 & 16 & 32 \\ \text { Beam FWHM: } & 0.896 \times 0.486\end{array}\right)$

Clean RR map. Array: BFFHHKKMMNNOPSSTWW

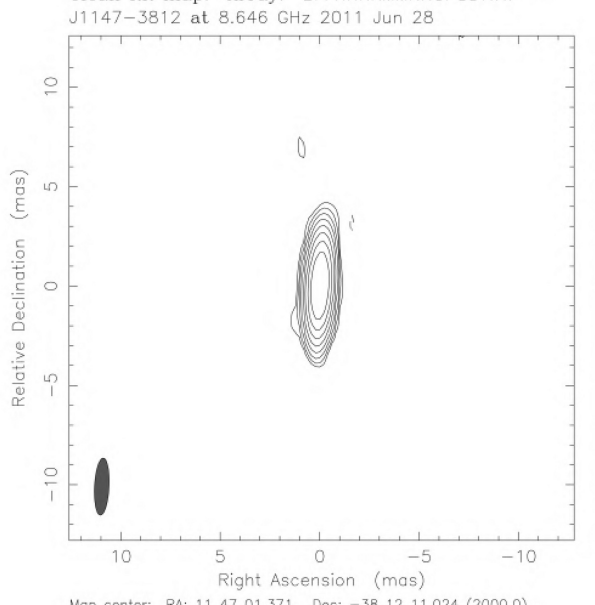

Map center: RA: 1147 01.371. Dec: -381211.024 (2000.0)

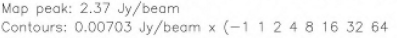
Contours: 128)

Beam FWHM: $2.87 \times 0.733$ (mos) at $-2.95^{\circ}$

Clean RR map. Array: VLBA Correlator

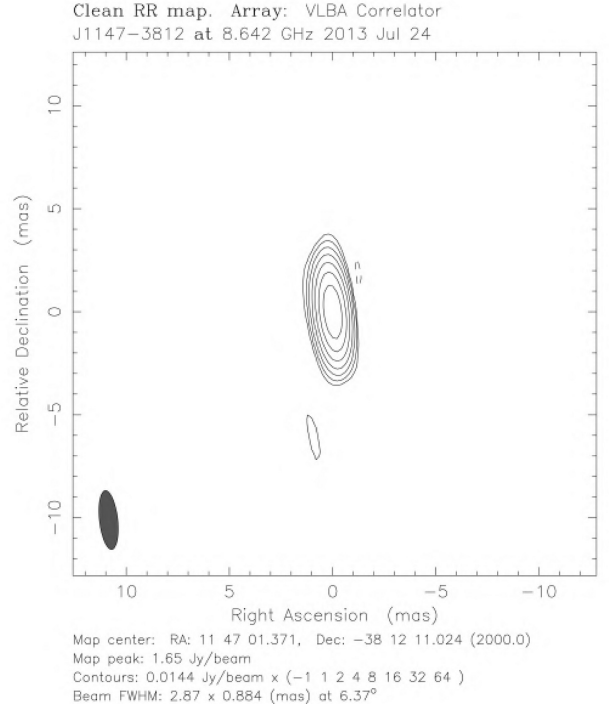

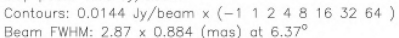

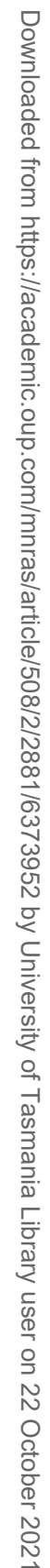

Figure B1 - continued

MNRAS 508, 2881-2896 (2021) 

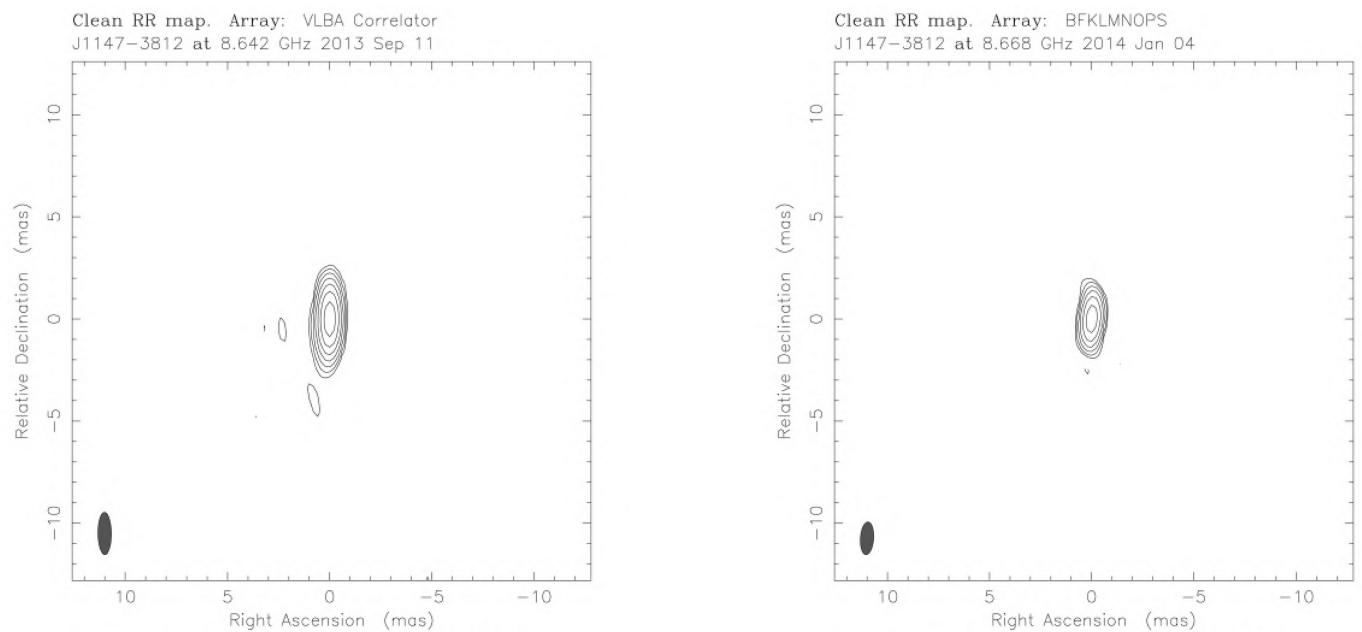

Map center: FA: 1147 01.371, Dec: -381211.024 (2000.0)

Map peok: 1.52 Jy/beom

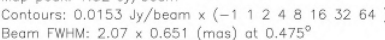

Clean RR map. Array: VLBA Correlator

J1147-3812 at $8.652 \mathrm{GHz} 2014$ Aug 09

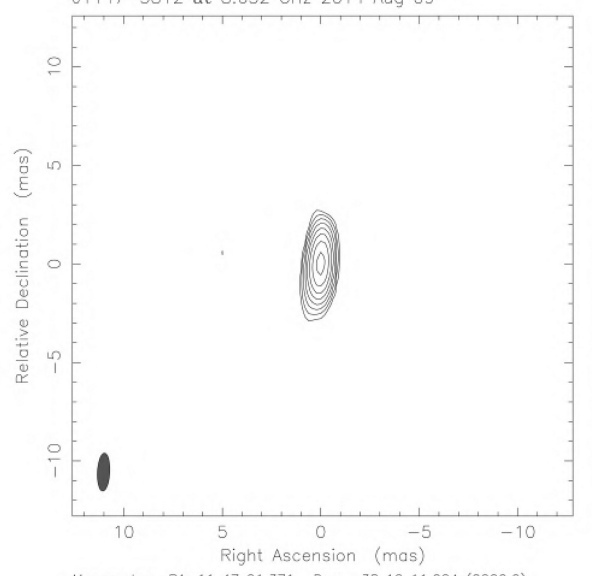

Nap center: RA: 1147 01.371, Dec: -38 1211.024 (2000,0)

Map peak: $1.57 \mathrm{Jy} / \mathrm{beam}$

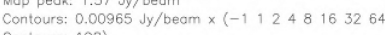

Beam FwHil 1.93 x

Clean RR map. Array: BFHKLMNOPS

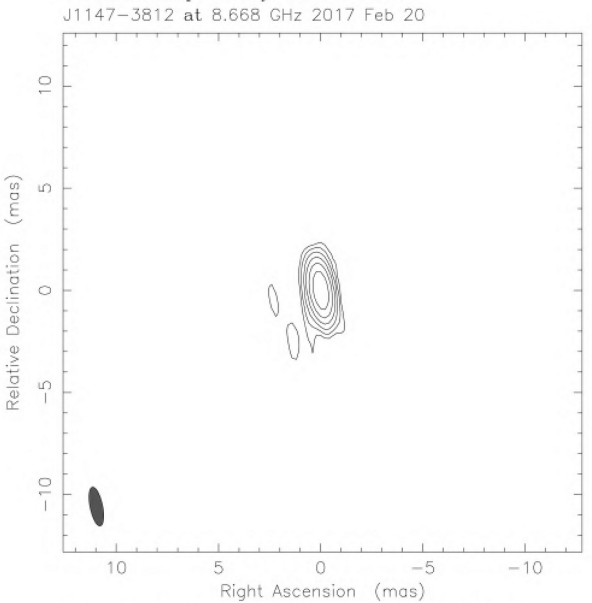

Right Ascension (mas)

Map center: RA: 1147 01.371, Dec: -38 1211.024 (2000.0)

Map peak: $0.995 \mathrm{Jy} / \mathrm{beam}$

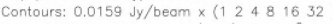

Beam FWHM: $1.94 \times 0.616$ (mas) at $11.5^{\circ}$

47 01.371, Dec: $-381211.024(2000.0)$

Map center: RA: 1147 01.371, Dec: -381211

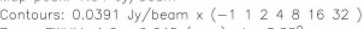

Beam FWHM: $1.6 \times 0.645$ (mas) ot $-3.88^{\circ}$

Clean RR map, Array: BFHLMNOPS

J1147-3812 at $8.668 \mathrm{GHz} 2017$ Jan 22

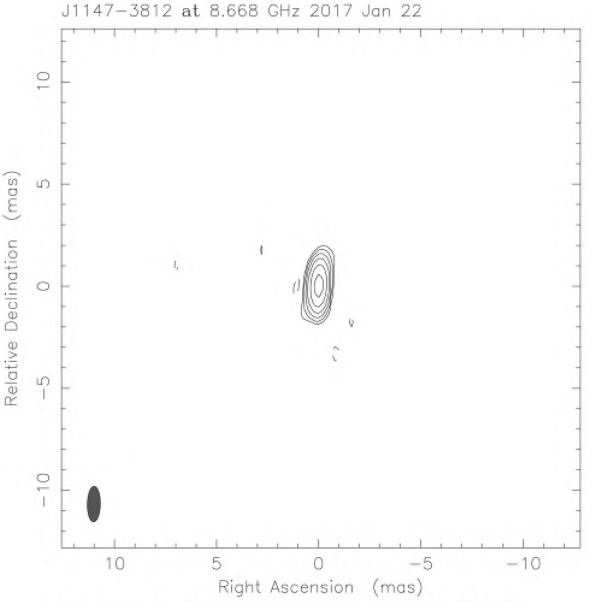

Map center: RA: 1147 01.371. Dec: -381211.024 (2000.0)

Map peak: $0.906 \mathrm{Jy} / \mathrm{beam}$

112481632

Clean RR map. Array: BFHKLMNOPS

J1147-3812 at $8.668 \mathrm{GHz} 2017$ Feb 25

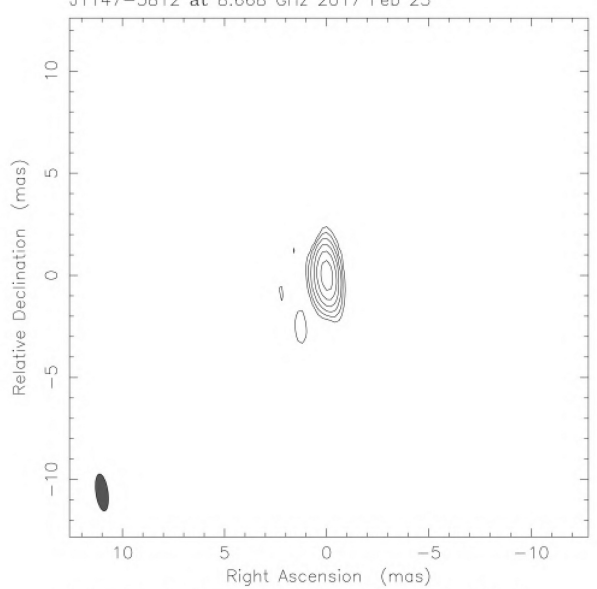

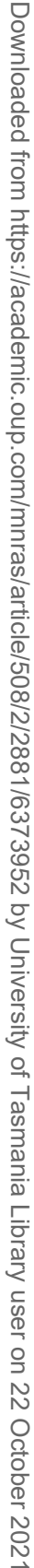

Map center: RA: 1147 01.371, Dec: -38 1211.024 (2000.0)

Map peok: $0.963 \mathrm{Jy} /$ beam

Contours: $0.0194 \mathrm{Jy} / \mathrm{beam} \times\left(\begin{array}{llllll}1 & 2 & 4 & 8 & 16 & 32\end{array}\right)$

Beam FWHM: $1.83 \times 0.599$ (mas) at $7.93^{\circ}$

Figure B1 - continued 
Clean RR map. Array: BFHKLMNOPS

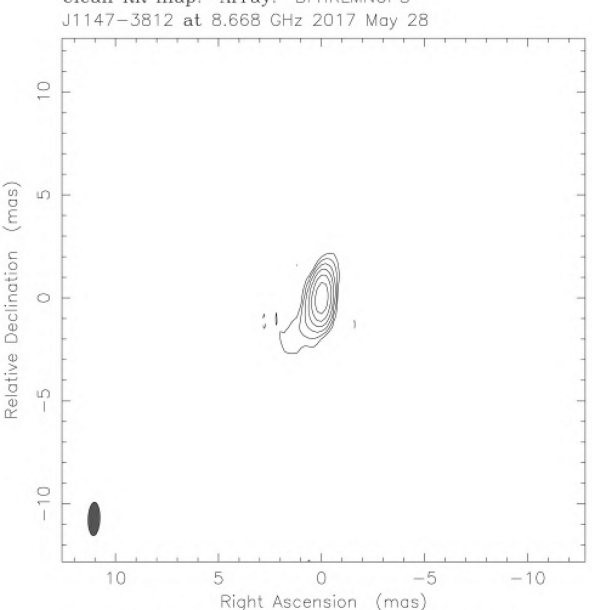

Map center: RA: 114701.371 . Dec: $-381211.024(2000.0)$

Contours: $0.0193 \mathrm{Jy} /$ beom $\times(-1) 12481632)$

Beam FWHM: $1.62 \times 0.573$ (mas) at -2.010

Clean RR map. Array: BFHKLMNOPS

J1147-3812 at $8.668 \mathrm{GHz} 2017$ Jun 16

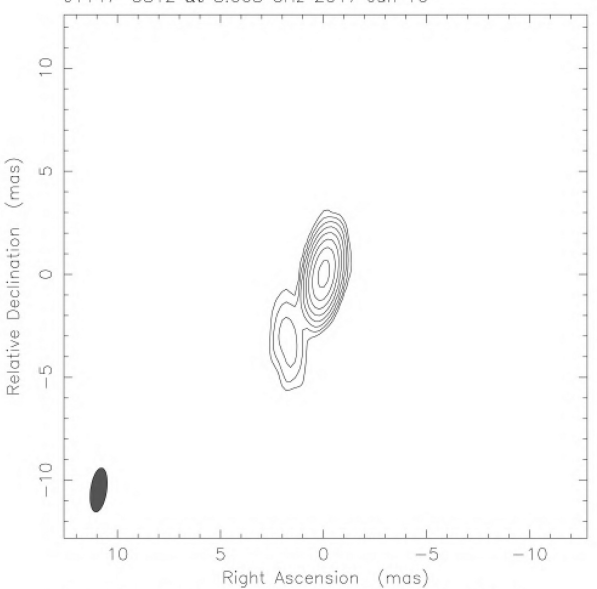

Map center: RA: 1147 01.371, Dec: - 38 12 11.024 (2000.0)

Map peak: $1.24 \mathrm{Jy} /$ beam

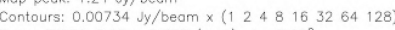

Beam FWHM: $2.15 \times 0.785$ (mas) at $-8.12^{\circ}$

Clean RR map Array: BFHKLMNOPS

$11147-3812$ at $8.668 \mathrm{GHz} 2017 \mathrm{Jul} \mathrm{O9}$

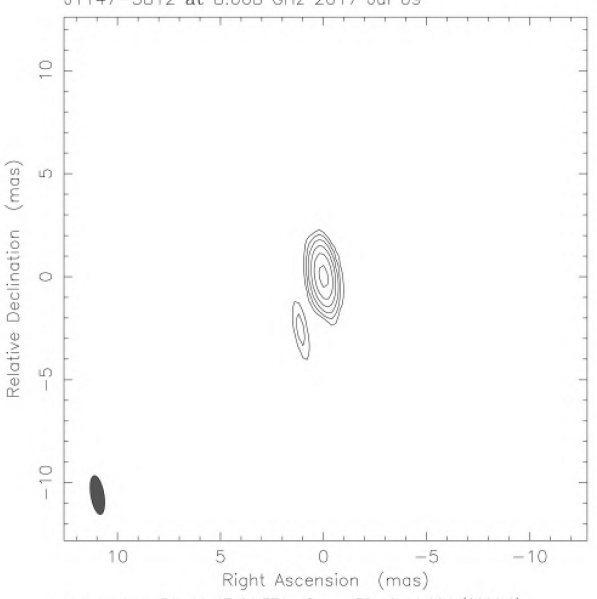

Map center: RA: 1147 01.371, Dec: -381211.024 (2000.0)

Map peak: $1.44 \mathrm{Jy} /$ beam

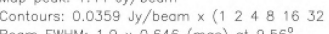

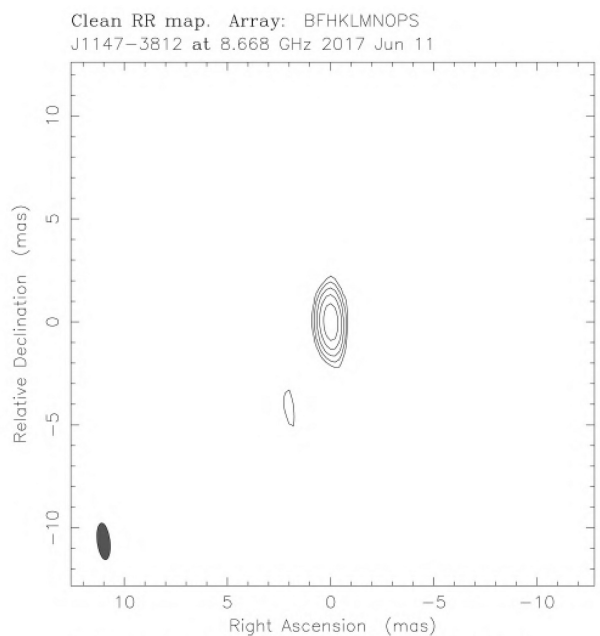

Map center: RA: 114701.371 , Dec: -381211.024 (2000.0)

Contours: $0.0311 \mathrm{Jy} / \mathrm{becm} \times(124816)$

Beam FWHM= 178 × 0.615 (mas) at $6.73^{\circ}$

Clean RR map. Array. VLBA Correlator

J1147-3812 at $8.636 \mathrm{GHz} 2017$ Jun 28

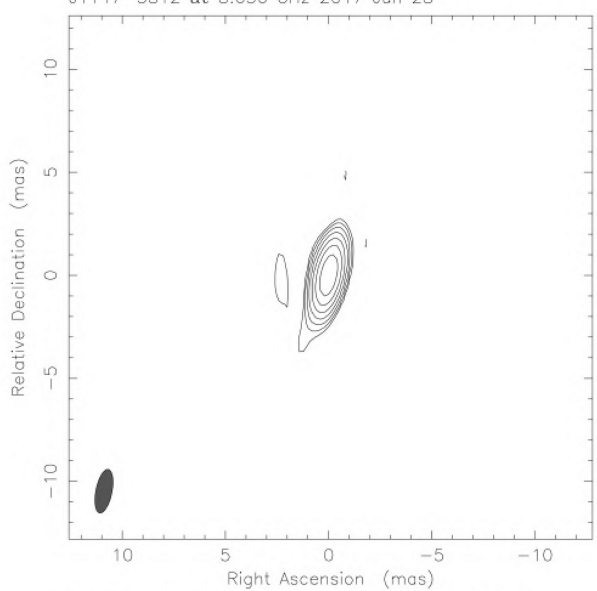

Nep center: PA: 114701.371 . Deci - 381211.024 (2000.0)

Map peak: $1.38 \mathrm{Jy} /$ beam

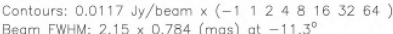

$\times 0.784$ (mas) at -11.30

Clean RR map. Array: BFHKLMNOPS

J1147-3812 at $8.668 \mathrm{GHz} 2017$ Jun 16

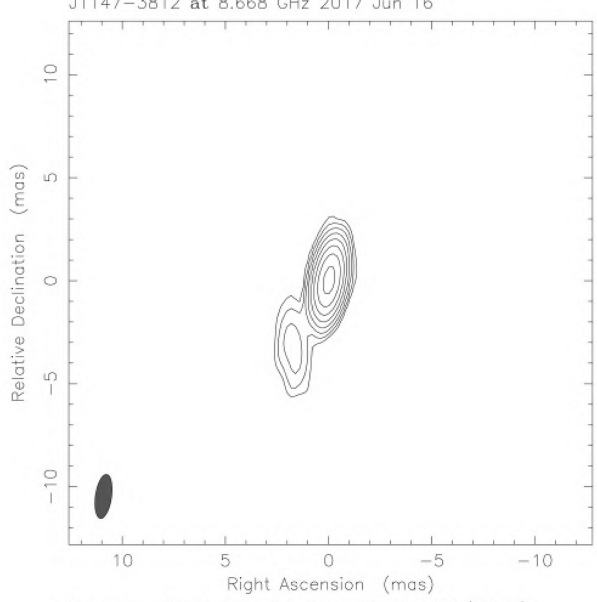

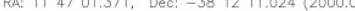

Map peak: $1.24 \mathrm{Jy} /$ beam

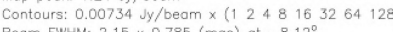

Figure B1 - continued 

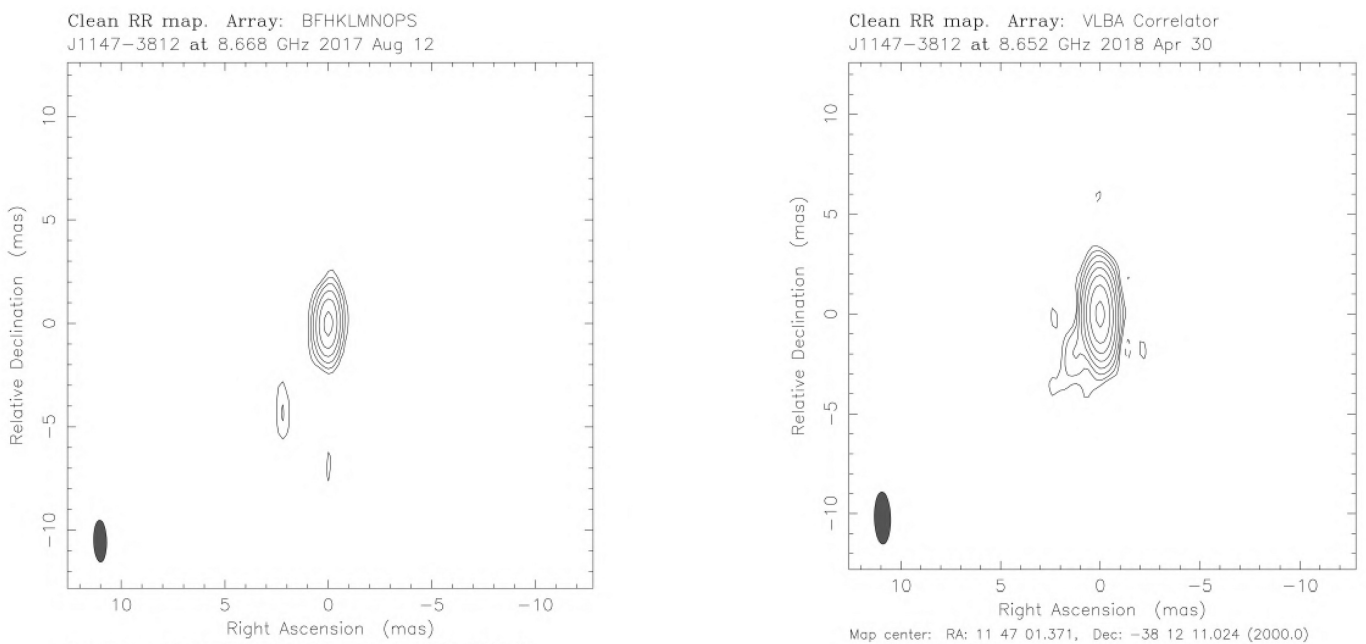

Mop center: RA: 1147 01.371, Dec: -381211.024 (2000.0)

(1)

0.626 (mas) at 1.41

Clean RR map. Array: VLBA Correlator

J1147-3812 at $8.652 \mathrm{GHz} 2018$ Nov 03

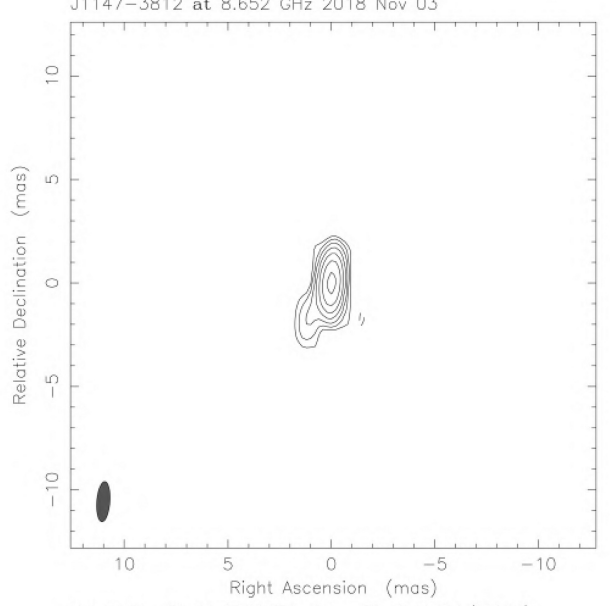

Map center: RA: 114701.371 , Dec: $-381211.024(2000.0)$ Map peak: $1.13 \mathrm{Jy} /$ beam

$x(-1) 248163264$

Beam FWHM: $2.62 \times 0.815$ (mos) ot $1.26^{\circ}$

Clean RR map. Array: VLBA Correlator

J1147-3812 at $8.652 \mathrm{GHz} 2018 \mathrm{Dec} 14$

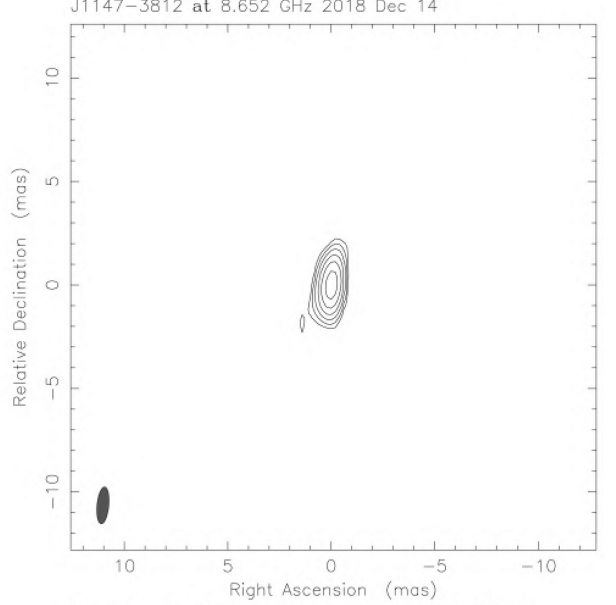

Map center: RA: 114701.371 , Dec: $-381211.024(2000.0)$
Map peak: $0.98 \mathrm{Jy} /$ beam

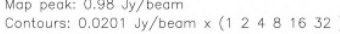

Beam FWHM: $1.78 \times 0.567$ (mas) at $-5.47^{\circ}$

This paper has been typeset from a $\mathrm{T}_{\mathrm{E}} \mathrm{X} / \mathrm{LAT}_{\mathrm{E}} \mathrm{X}$ file prepared by the author.

Figure B1 - continued 